\title{
Analytical and numerical study of travelling waves using the Maxwell-Cattaneo relaxation model extended to reaction-advection-diffusion systems
}

\author{
V. A. Sabelnikov, ${ }^{1, *}$ N. N. Petrova, ${ }^{1}$ and A. N. Lipatnikov ${ }^{2, \dagger}$ \\ ${ }^{1}$ ONERA-The French Aerospace Laboratory, F-91761 Palaiseau, France \\ ${ }^{2}$ Department of Applied Mechanics, Chalmers University of Technology, Gothenburg 412-96, Sweden
}

(Received 11 February 2016; published 21 October 2016)

\begin{abstract}
Within the framework of the Maxwell-Cattaneo relaxation model extended to reaction-diffusion systems with nonlinear advection, travelling wave (TW) solutions are analytically investigated by studying a normalized reaction-telegraph equation in the case of the reaction and advection terms described by quadratic functions. The problem involves two governing parameters: (i) a ratio $\varphi^{2}$ of the relaxation time in the Maxwell-Cattaneo model to the characteristic time scale of the reaction term, and (ii) the normalized magnitude $N$ of the advection term. By linearizing the equation at the leading edge of the TW, (i) necessary conditions for the existence of TW solutions that are smooth in the entire interval of $-\infty<\zeta<\infty$ are obtained, (ii) the smooth TW speed is shown to be less than the maximal speed $\varphi^{-1}$ of the propagation of a substance, (iii) the lowest TW speed as a function of $\varphi$ and $N$ is determined. If the necessary condition of $N>\varphi-\varphi^{-1}$ does not hold, e.g., if the magnitude $N$ of the nonlinear advection is insufficiently high in the case of $\varphi^{2}>1$, then, the studied equation admits piecewise smooth TW solutions with sharp leading fronts that propagate at the maximal speed $\varphi^{-1}$, with the substance concentration or its spatial derivative jumping at the front. An increase in $N$ can make the solution smooth in the entire spatial domain. Moreover, an explicit TW solution to the considered equation is found provided that $N>\varphi$. Subsequently, by invoking a principle of the maximal decay rate of TW solution at its leading edge, relevant TW solutions are selected in a domain of $(\varphi, N)$ that admits the smooth TWs. Application of this principle to the studied problem yields transition from pulled (propagation speed is controlled by the TW leading edge) to pushed (propagation speed is controlled by the entire TW structure) TW solutions at $N=N_{\mathrm{cr}}=\sqrt{1+\varphi^{2}}$, with the pulled (pushed) TW being relevant at smaller (larger) $N$. An increase in the normalized relaxation time $\varphi^{2}$ results in increasing $N_{\mathrm{cr}}$, thus promoting the pulled TW solutions. The domains of $(\varphi, N)$ that admit either the smooth or piecewise smooth TWs are not overlapped and, therefore, the selection problem does not arise for these two types of solutions. All the aforementioned results and, in particular, the maximal-decay-rate principle or appearance of the piecewise smooth TW solutions, are validated by numerically solving the initial boundary value problem for the reaction-telegraph equation with natural initial conditions localized to a bounded spatial region.
\end{abstract}

DOI: 10.1103/PhysRevE.94.042218

\section{INTRODUCTION}

Propagation of a wave in a nonequilibrium medium is a widespread phenomenon relevant to many branches of science, such as biology [1-5], economics [6], combustion [7,8], chemistry [9], thermoelasticity and the thermal convection in nanofluids [10], physics [11,12], meteorology, oceanology and hydrogeology [13-15], etc. Mathematical models of the phenomenon are based on a scalar transport partial differential equation (PDE), which reads

$$
\frac{\partial u}{\partial t}+\frac{\partial j}{\partial x}=w(u)
$$

in the one-dimensional case. Here, $u(x, t)$ is the concentration of a substance (or population), $j(x, t)$ is the substance flux across $x$ at time $t$, and $w(u)$ is a rate function (source). In the literature, various constitutive relations between $j(x, t)$ and $u(x, t)$ were applied to various problems. The goal of the present work is to study traveling wave (TW) solutions $u(z)$ to the scalar balance Eq. (1) supplemented with the following

\footnotetext{
*vladimir.sabelnikov@onera.fr

†lipatn@chalmers.se
}

generic constitutive relation:

$$
\frac{\partial j}{\partial t}+v^{2} \frac{\partial u}{\partial x}=-\frac{1}{\tau}(j-q) .
$$

Here, $z=x-S t, S$ is TW speed, the flux $q=q(u)$ depends solely on $u, v^{2} \tau=D$, and both the relaxation time $\tau$ and diffusion coefficient $D$ are considered to be constant in homogeneous space. If the concentration is nondimensional and $x$ is distance, then, $j, v$, and $q$ have dimension of velocity. The terms of Eq. (2) are introduced for the following three reasons.

First, if the nonlinear advection flux $q$ vanishes and the relaxation time is asymptotically short, i.e., $\tau \rightarrow 0$ with $\nu^{2} \tau=$ $D=$ const, then, Eq. (2) reduces to Fick's law of diffusion. This phenomenological law is widely used in various fields of science [16].

Second, the nonlinear term $q(u)$ allows for deterministic motion of substance in (or opposite to) the direction of its gradient, in addition to the Brownian motion modeled by the second term on the left hand side (LHS). Such deterministic motion is relevant to various phenomena in biology, physiology, chemistry, etc. [3,4,17]. For instance, a nonlinear advection term is invoked [18-21] to model the so-called countergradient scalar transport in turbulent 
flows. Such a transport is well documented, e.g., in premixed turbulent flames [22-24], where it is driven by preferential acceleration of lighter (when compared to unburned gas) combustion products by pressure gradient induced due to thermal expansion [25,26].

Third, the use of Fick's law, i.e., substitution of Eq. (2) with $\tau \rightarrow 0$ and $q=0$ into Eq. (1), is well known to yield infinitely high velocity of the substance propagation in the simplest case of $w \equiv 0$, e.g. $[16,27,28]$. To limit the propagation speed, Eq. (2) invokes a widespread model of the substance dispersion, developed by Maxwell [29] and Cattaneo [30,31], who introduced the following relaxation equation for the flux:

$$
\frac{\partial j}{\partial t}=-\frac{1}{\tau}\left(j-j_{\mathrm{eq}}\right),
$$

where the equilibrium flux $j_{\text {eq }}$ is given by Fick's law, i.e., $j_{\text {eq }}=j_{D} \equiv-D \partial u / \partial x$. The Maxwell-Cattaneo (MC) model, i.e., Eq. (1) with $w \equiv 0$ and Eq. (3), takes into account memory effects in the evolution of the substance flux, because $j$ approaches its equilibrium value $j_{\text {eq }}$ during a finite relaxation time $\tau$. The MC model results in the well-known telegraph (or damped wave) equation, which is widely used in physics and biology, as reviewed in detail elsewhere [28]. Accordingly, the MC model yields a finite speed of the substance propagation, whose magnitude is limited by $v$. Indeed, if the initial conditions are compact, i.e.,

$$
\begin{aligned}
& u \equiv 1, \quad j \equiv 0, \quad x<x_{10}, \\
& 0<u<1, \quad j \neq 0, \quad x_{10}<x<x_{20}, \\
& u \equiv 0, \quad j \equiv 0, \quad x>x_{20},
\end{aligned}
$$

at $t=0$, then, the domain of the influence of the initial conditions

$$
x_{10}-v t=x_{l}(t)<x<x_{r}(t)=x_{20}+v t
$$

is bounded by left and right sharp fronts, $x_{l}(t)$ and $x_{r}(t)$, respectively. The concentration $u$ and flux $j$ are not perturbed outside this domain.

Thus, Eqs. (1) and (2) allow for (i) substance creation, e.g., in chemical reactions, (ii) Fick's diffusion, e.g., due to Brownian motion, (iii) memory effects in the development of the flux $j$ (the MC model), and (iv) nonlinear advection, e.g., chemotaxis (production of chemicals by population organisms) in biology [17] or countergradient transport in turbulent flames [22-24].

Accordingly, Eq. (2) subsumes various widely used constitutive relations between $j(x, t)$ and $u(x, t)$. For instance, first, if $q=0$ and $\tau \rightarrow 0$ with $\nu^{2} \tau=D=$ const, then, Eqs. (1) and (2) reduce to a well-known reaction-diffusion equation, which is widely applied in various fields of science [32]. Second, if $q=k u(1-u)$ is finite (any sign of the coefficient $k$ is admitted), but $\tau \rightarrow 0$, then, Eqs. (1) and (2) reduce to a wellknown convection-reaction-diffusion equation introduced into mathematical biology and investigated by Murray [3,4]. Third, if $\tau$ is finite, but both $q$ and the rate $w$ vanish, then Eqs. (1) and (2) model turbulent diffusion of an admixture in a flow [33]. Fourth, if $q$ vanishes, but both $\tau$ and $w$ are finite, then Eqs. (1) and (2) extend the MC model to chemically reacting substance and this particular problem is discussed in detail elsewhere [16,34-36]. Fifth, if both $q$ and $\tau$ are finite, but $w$ vanishes, then, Eqs. (1) and (2) reduce to a model of traffic flow, developed by Jordan [37] who considered $q(u)$ to be a quadratic function of the traffic density $u(x, t)$.

Nevertheless, despite that Eqs. (1) and (2) subsume various known models of various phenomena, as illustrated above, the present authors are not aware of a study of these two equations in a general case of finite $w, q$, and $\tau$. The present work aims at filling this gap and investigating the interplay of all three effects (reaction, nonlinear advection, and memory) by (i) exploring TW solutions to Eqs. (1) and (2) and (ii) describing selection of relevant TW solutions. We consider a TW solution to be relevant if, at $t \rightarrow \infty$, it is reached by a solution to initial boundary value problem (IBVP) stated by Eqs. (1) and (2) with compact initial conditions given by Eq. (4) and with the following boundary conditions for a substance concentration $u$ :

$$
u(-\infty, t)=1, u(\infty, t)=0 .
$$

Here, for determinacy, we address a wave that propagates from left to right. Moreover, obtained analytical results will be validated by numerically solving the stated IBVP. The generic Eqs. (1) and (2) are relevant, e.g., to modeling of premixed turbulent burning. In this particular case, $u$ is a mean combustion progress variable [22], $w$ is a mean rate of product creation, the second term on the LHS of Eq. (2) models turbulent diffusion, and the nonlinear advection term is associated with pressure-driven countergradient transport discussed above. In the case of $\tau \rightarrow 0$ and constant $\nu^{2} \tau=D$, Eqs. (1) and (2) were already applied to modeling premixed turbulent burning [19-21]. However, in this case, Eqs. (1) and (2) admit an infinitely high speed of the propagation of flame leading edge, whereas, from the physical viewpoint, the propagation speed should be limited by the rms flow velocity in intense turbulence [7,38,39]. The use of the MC model, i.e., a study of Eq. (2) with a finite $\tau$, allows us to overcome this difficulty and to explore flame propagation at a finite speed. Accordingly, a particular goal of this work is to study the influence of the memory effects (finite values of $\tau$ ) on turbulent flame speed and transition from pulled to pushed flames. It is worth noting that while combustion is accompanied be density variations, they are not addressed in the present work.

The rest of the paper is organized as follows. In Sec. II, an IBVP associated with Eqs. (1) and (2) is stated in a nondimensional form and a method of analysis is outlined. In Sec. III, a boundary value problem (BVP) resulting from the stated IBVP is addressed by studying various types of TW solutions to the BVP and finding boundaries of different regimes of TW propagation as functions of relative magnitudes of various terms in Eqs. (1) and (2). In Sec. IV, an explicit smooth pushed TW solution is obtained. In Sec. V, the relevant TW solutions to the stated IBVP are addressed. The obtained analytical results are supported by numerical simulations of the IBVP in Sec. VI, followed by conclusions.

\section{STATEMENT OF THE PROBLEM}

\section{A. Governing equations}

For dimensional reasons,

$$
w(u)=\frac{\omega(u)}{\tau_{f}}, \quad q(u)=-V Q(u),
$$


where $\tau_{f}$ is a characteristic time scale of the source term, $-\infty<V<\infty$ is a dimensional $(\mathrm{m} / \mathrm{s})$ input parameter that controls the intensity of the nonlinear advection, while the functions $\omega(u)$ and $Q(u)$ are dimensionless.

In the present study, we consider the case of (i) a monostable source term $\omega(u)$, i.e., it has simple zeros at $u=0$ and $u=1$, but is positive between,

$$
\omega(0)=\omega(1)=0, \quad \omega(0<u<1)>0
$$

and (ii) an advection that vanishes at $u=0$ and $u=1$, but is positive between, i.e.,

$$
Q(0)=Q(1)=0, \quad Q(0<u<1)>0 .
$$

In such a case, $u=0$ and $u=1$ are homogeneous unstable and stable states, respectively.

Normalization of Eqs. (1), (2), and (7) using the length $\sqrt{D \tau_{f}}$ and time $\tau_{f}$ scales yields

$$
\begin{gathered}
\frac{\partial u}{\partial \theta}+\frac{1}{\varphi} \frac{\partial \hat{j}}{\partial \xi}=\omega, \\
\frac{\partial \hat{j}}{\partial \theta}+\frac{1}{\varphi} \frac{\partial u}{\partial \xi}=-\frac{1}{\varphi^{2}} \hat{j}-\frac{1}{\varphi} N Q(u),
\end{gathered}
$$

respectively. Here, $\xi=x / \sqrt{D \tau_{f}}, \theta=t / \tau_{f}, \hat{j}=\varphi j / \sqrt{D / \tau_{f}}$, $\varphi^{2}=\tau / \tau_{f}, N=V / \sqrt{D / \tau_{f}}, \varphi>0,-\infty<N<\infty$, and the same symbol $u$ is used for the substance concentration as a function of dimensionless variables $\xi$ and $\theta$. Initial and boundary conditions are given by normalized Eqs. (4) and (5), respectively.

The stated problem involves two nondimensional input parameters: (i) the normalized magnitude $N$ of the nonlinear advection flux (in the case of premixed turbulent combustion, $N$ is associated with a widely used Bray number [18,22]), and (ii) a ratio $\varphi^{2}$ of the relaxation time in the MC model to the characteristic time scale of the source term $w$ on the right hand side (RHS) of Eq. (1). The inverse ratio of the two time scales controls the maximal wave propagation speed, i.e., $v / \sqrt{D / \tau_{f}}=v / \sqrt{v^{2} \tau / \tau_{f}}=\varphi^{-1}$. In the case of $\varphi<1$ (or $\varphi>1$ ), the relaxation time scale is shorter (longer) than the characteristic reaction time scale. In the case of $N<1$ (or $N>1$ ), the magnitude of Fik's diffusion flux is larger (smaller) than the magnitude of the nonlinear advection flux. If applied to premixed combustion, the case of $N<1$ (or $N>1$ ) is associated with gradient (countergradient) turbulent scalar transport.

Differentiating Eqs. (10) and (11) with respect to $\xi$ and $\theta$, respectively, subtracting the latter equation from the former, and using Eq. (10) to exclude the spatial derivative of the flux, Eqs. (10) and (11) can be recast into the following reactiontelegraph equation:

$$
\varphi^{2} \frac{\partial^{2} u}{\partial \theta^{2}}+\left(1-\varphi^{2} \frac{d \omega}{d u}\right) \frac{\partial u}{\partial \theta}-N \frac{d Q}{d u} \frac{\partial u}{\partial \xi}=\frac{\partial^{2} u}{\partial \xi^{2}}+\omega(u),
$$

which does not involve the flux.

\section{B. Riemann invariants and maximal propagation speed}

Indeed, using the method described, e.g., in [27,40], the hyperbolic Eq. (12) can be recast into the characteristic form

$$
\begin{aligned}
& \frac{\partial u_{+}}{\partial \theta}+\frac{1}{\varphi} \frac{\partial u_{+}}{\partial \xi}=-\frac{1}{2 \varphi^{2}}\left(u_{+}-u_{-}\right)+\frac{\omega}{2}-\frac{N Q}{2 \varphi}, \\
& \frac{\partial u_{-}}{\partial \theta}-\frac{1}{\varphi} \frac{\partial u_{-}}{\partial \xi}=-\frac{1}{2 \varphi^{2}}\left(u_{+}-u_{-}\right)+\frac{\omega}{2}+\frac{N Q}{2 \varphi},
\end{aligned}
$$

where $u_{+}$and $u_{-}$are Riemann invariants, e.g., [27,40], defined as follows:

$$
\begin{gathered}
u_{+}=\frac{1}{2}(u+\hat{j}), \quad u_{-}=\frac{1}{2}(u-\hat{j}), \\
u=u_{+}+u_{-}, \quad \hat{j}=u_{+}-u_{-}
\end{gathered}
$$

The hyperbolic PDEs (13) and (14) can be solved by integrating the following linear ordinary differential equations (ODEs) for the characteristic curves, e.g., [27,40]

$$
\begin{gathered}
\frac{d \xi_{ \pm}}{d \theta}= \pm \frac{1}{\varphi} \\
\frac{d_{+} u_{+}}{d \theta}=\frac{\partial u_{+}}{\partial \theta}+\frac{1}{\varphi} \frac{\partial u_{+}}{\partial \xi}=-\frac{1}{2 \varphi^{2}}\left(u_{+}-u_{-}\right)+\frac{\omega}{2}-\frac{N Q}{2 \varphi}
\end{gathered}
$$

$$
\frac{d_{-} u_{-}}{d \theta}=\frac{\partial u_{-}}{\partial \theta}-\frac{1}{\varphi} \frac{\partial u_{-}}{\partial \xi}=-\frac{1}{2 \varphi^{2}}\left(u_{-}-u_{+}\right)+\frac{\omega}{2}+\frac{N Q}{2 \varphi} .
$$

It should be noted that the characteristic lines $\xi_{ \pm}(\theta)$, described by Eq. (16), are uncoupled from Eqs. (17) and (18) for the Riemann invariants, which remain constant along the corresponding characteristic line if terms on the RHSs of Eqs. (17) and (18) vanish. In particular, the RHSs of Eqs. (17) and (18) vanish along characteristics lines that originate from $\xi_{-}(0) \leqslant \xi_{10}=x_{10} / \sqrt{D \tau_{f}}$ and $\xi_{+}(0) \geqslant \xi_{20}=$ $x_{20} / \sqrt{D \tau_{f}}$, where the boundaries $x_{10}$ and $x_{20}$ stem from the initial conditions given by Eq. (4). Therefore, the domain of the influence of these initial conditions is as follows:

$$
\xi_{10}-\varphi^{-1} \theta=\xi_{l}(\theta)<\xi<\xi_{r}(\theta)=\xi_{20}+\varphi^{-1} \theta, \quad \theta>0 .
$$

It is bounded by sharp left and right fronts, $\xi_{l}(\theta)$ and $\xi_{r}(\theta)$, respectively, which are nothing but the trailing and leading characteristic lines, respectively, that emanate from the left and right edges, respectively, of the compact domain of the initial conditions given by the normalized Eq. (4). Beyond this domain, the concentration $u$ and flux $\hat{j}$ are not perturbed, i.e.,

$$
\begin{array}{ll}
u(\xi, \theta) \equiv 1, \quad \hat{j}(\xi, \theta) \equiv 0, \quad \xi<\xi_{l}(\theta)=\xi_{10}-\varphi^{-1} \theta, \\
u(\xi, \theta) \equiv 0, \quad \hat{j}(\xi, \theta) \equiv 0, \quad \xi>\xi_{r}(\theta)=\xi_{20}+\varphi^{-1} \theta, \theta>0 .
\end{array}
$$

\section{Method of research}

In the rest of the present paper, we will restrict ourselves to a particular but typical case of $\omega(u)=u(1-u)$ and $Q(u)=$ $u(1-u)$. 
In the case of $\varphi=0$, i.e., the lack of memory effects, Eq. (12) reduces to the following reaction-diffusion equation:

$$
\frac{\partial u}{\partial \theta}-N Q^{\prime}(u) \frac{\partial u}{\partial \xi}=\frac{\partial^{2} u}{\partial \xi^{2}}+\omega(u) .
$$

If, moreover, the advection term is absent, i.e., $N=0$, and $\omega=u-u^{q}$ with $q>1$, then, Eq. (21) reduces to a parabolic reaction-diffusion PDE known as the Fisher-KPP equation in honor of a pioneering work by Fisher [1] and Kolmogorov, Petrovsky, and Piskounov [2]. The rigorous mathematical theory of the KPP equation supplemented with the initial and boundary conditions given by normalized Eqs. (4) and (5) is well elaborated in Ref. [2] and in subsequent studies reviewed elsewhere [3-6,12,32]. A number of important, but mathematically less rigorous, results were also obtained by investigating an IBVP stated by Eqs. (4), (5), and (21), as discussed in detail elsewhere [3-6,12,32]. It is also worth noting that there are other methods of studying the Fisher-KPP equation, e.g., the singular perturbation approach [41,42], which will not be used in the present work.

Accordingly, in Secs. III-V, we will assume that certain analytical results obtained by studying the two aforementioned problems can also be applicable to an IBVP posed by Eqs. (4), (5), and (10) and (11) or (12). Because expressions obtained by drawing such an analogy between Eqs. (12) and (21) require validation, a numerical study of unsteady solutions to Eqs. (4), (5), (10), and (11) was also performed and results will be discussed in Sec. VI.

In particular, the following results [2-6,12,32] obtained by investigating the KPP equation and Eq. (21) will be invoked by us.

First, there is a continuous spectrum of smooth TW solutions $u(\xi, \theta)=U(\zeta)$, where $\zeta=\xi-\Lambda \theta$ is a wave variable and $\Lambda$ designates the wave speed, to a boundary value problem (BVP) stated by the TW counterpart of the KPP equation and the boundary conditions given by Eq. (5), with the TW speeds being bounded from below, i.e., $\Lambda \geqslant \Lambda_{K P P}=2$ [2].

Second, the lowest TW speed $\Lambda_{K P P}=2$ can be found by linearizing the KPP equation at the wave leading edge, i.e., at $u \rightarrow 0$ [2].

Third, the linearized KPP equation admits TW solutions that move at the same speed $\Lambda$, but have two different decay rates $\kappa=(d U / d \zeta)_{u \rightarrow 0}$, i.e., $\kappa_{+}(\Lambda)$ and $\kappa_{-}(\Lambda)$, with $\kappa_{+}(\Lambda) \geqslant$ $\kappa_{-}(\Lambda)$ [2]. However, this does not mean that the nonlinear KPP equation admits two different TW solutions that move at the same speed $\Lambda$, i.e., a solution to the linearized equation is not always a solution to the nonlinear KPP equation.

Fourth, as $\theta \rightarrow \infty$, a time-dependent solution to the KPP IBVP approaches the TW solution characterized by the slowest propagation speed $\Lambda_{K P P}=2$ provided that the initial wave profile is steep enough [2], e.g., is described with the initial conditions given by normalized Eq. (4). Such a TW is called pulled wave [12] in order to stress that its speed can be found by linearizing the KPP equation at $u \rightarrow 0$, i.e., the wave is pulled by its leading edge.

Fifth, the relevant TW solution to the KPP IBVP features $\kappa_{+}\left(\Lambda_{K P P}\right)=\kappa_{-}\left(\Lambda_{K P P}\right)[2]$ and is characterized not only with the lowest propagation speed, but also with the highest decay rate $\kappa_{-}\left(\Lambda_{K P P}\right)$ that is consistent with the nonlinear KPP equation [2]. The point is that (i) $\kappa_{-}(\Lambda)$ is decreased when $\Lambda$ is increased, but (ii) the branch $\kappa_{+}(\Lambda)$ of solutions to the linearized KPP equation does not satisfy the nonlinear KPP equation if $\Lambda>\Lambda_{K P P}=2$ [2].

Sixth, under certain conditions, the BVP associated with Eqs. (5) and (21) admits the so-called pushed TW solutions [12] whose speed $\Lambda_{p}$ cannot be determined by linearizing Eq. (21) at $u \rightarrow 0$, e.g., because either the linear analysis admits all $\Lambda>0$ or TW solutions to the linearized Eq. (21) do not satisfy the nonlinear Eq. (21) if $\Lambda<\Lambda_{p}$. The decay rate of such a pushed TW belongs to the $\kappa_{+}(\Lambda)$ branch obtained by linearizing Eq. (21) at $u \rightarrow 0$. In other words, for such pushed TW solutions, the spectrum $\Lambda(\kappa)$ consists only of an isolated discrete point $\Lambda=\Lambda_{p}\left(\kappa_{+}\right)$.

Seventh, if (i) the linearized Eq. (21) admits TW solutions with $0<\Lambda_{l} \leqslant \Lambda \leqslant \Lambda_{p}$, (ii) there is a pushed TW solution to the nonlinear Eq. (21), and (iii) the decay rate $\kappa_{+}\left(\Lambda_{p}\right)$ is higher than decay rates $\kappa_{-}\left(\Lambda_{l} \leqslant \Lambda<\Lambda_{p}\right)$ obtained from the analysis of the linearized Eq. (21), then, a time-dependent solution to the IBVP associated with Eq. (21) approaches the pushed TW solution characterized by the highest decay rate [12,32,43-45].

Based on the results cited above, the following study will consist of four steps.

In Sec. III, pulled TW solutions to the considered problem will be addressed and the TW counterparts of Eqs. (10) and (11) will be linearized at $u \rightarrow 0$ in order to determine (i) a domain of $(\varphi, N)$ such that a smooth (in entire interval $-\infty<$ $\zeta<\infty$ ) solution to the linearized equations exists and (ii) a domain of $(\varphi, N)$ such that $\Lambda_{\min }>0$ and $\Lambda_{\min }$ is lower than the maximal allowed propagation speed $\varphi^{-1}$. Moreover, because the relevant solution to the KPP IBVP is characterized with $\kappa_{+}\left(\Lambda_{K P P}\right)=\kappa_{-}\left(\Lambda_{K P P}\right)=\kappa_{m}\left(\Lambda_{K P P}\right)$ [2], the focus of our analysis will be placed on $\kappa_{m}\left(\Lambda_{\min }, \varphi, N\right)$ provided that $\Lambda_{\min }>$ 0 , rather than $\kappa_{+}\left(\Lambda>\Lambda_{\min }, \varphi, N\right)$ or $\kappa_{-}\left(\Lambda>\Lambda_{\min }, \varphi, N\right)$.

Then, analytical expressions for the piecewise smooth TWs with discontinuous $U$ or its derivative at the sharp leading front will be obtained. These TWs propagate at the maximal propagation speed $\varphi^{-1}$, i.e., $\Lambda=\varphi^{-1}$.

In Sec. IV, a particular explicit pushed TW solution to the studied IBVP will be found. The pushed TW is smooth at $-\infty<\zeta<\infty$ and propagates at a speed $\Lambda$ less than the maximal propagation speed $\varphi^{-1}$.

In Sec. V, the relevant smooth TW solutions will be selected between the pulled and pushed TWs based on two principles; (i) the lowest propagation speed [2] and (ii) the maximal decay rate [12,43-45]. First, when considering TW solutions to the linearized BVP, a TW solution that moves at the lowest speed $\Lambda_{\min }$ will be selected provided that $\Lambda_{\min }>0$. Second, a TW solution characterized by the highest decay rate will be selected by comparing solutions to the linearized BVP and a pushed solution to the nonlinear BVP. Selection between the smooth and piecewise smooth TWs will not be addressed, because the domains of $(\varphi, N)$ associated with the two types of TW solutions do not overlap.

In Sec. VI, the selected relevant TW solutions will be validated using numerical simulations.

A similar method of analysis was recently applied by us [19-21] to three different particular subclasses of the parabolic reaction-diffusion PDE (21), i.e., in the case of $\varphi=0$ in Eq. (12). 


\section{TW EQUATION AND BOUNDARY VALUE PROBLEM}

Substitution of a TW solution, i.e., a wave $u(\xi, \theta)=$ $U(\zeta), \hat{j}(\xi, \theta)=J(\zeta)$, where $\zeta=\xi-\Lambda \theta$, that propagates at a constant speed $\Lambda$ and has a stationary structure in the attached coordinate framework, into Eqs. (10) and (11) yields the following ODEs:

$$
\begin{gathered}
-\Lambda \frac{d U}{d \zeta}+\varphi^{-1} \frac{d J}{d \zeta}=\omega=U(1-U) \\
-\Lambda \frac{d J}{d \zeta}+\varphi^{-1} \frac{d U}{d \zeta}=-\varphi^{-2} J-\varphi^{-1} N U(1-U)
\end{gathered}
$$

Integration of Eq. (22) from $\zeta=-\infty$ to $\zeta=\infty$ results in the following expression for the TW speed $\Lambda$ :

$$
\Lambda=\int_{-\infty}^{\infty} \omega(u) d \zeta>0
$$

because $\hat{j}(-\infty, \theta)=\hat{j}(\infty, \theta)=0$. The integral on the RHS of Eq. (24) is positive for $\omega(U)>0$ and the TW speed is equal to the spatially integrated source term, as expected. The wave speed $\Lambda$ is unknown and has to be determined as a part of the solution to the problem.

Resolving Eqs. (22) and (23) with respect to $d U / d \zeta$ and $d J / d \zeta$ if $\Lambda \neq \varphi^{-1}$, we arrive at the following BVP for autonomous (independent of $\zeta$ ) system of two first-order nonlinear ODEs:

$$
\begin{gathered}
\frac{d U}{d \zeta}=\frac{-\Lambda \varphi^{2} U(1-U)+\varphi^{-1} J+N U(1-U)}{\Lambda^{2} \varphi^{2}-1}, \\
\frac{d J}{d \zeta}=\frac{\Lambda J+\Lambda \varphi N U(1-U)-\varphi U(1-U)}{\Lambda^{2} \varphi^{2}-1}, \\
U(-\infty)=1, \quad U(\infty)=0, \\
J(-\infty)=1, \quad J(\infty)=0 .
\end{gathered}
$$

Equations (25) and (26) can be rewritten in the phase space $(J, U)$,

$$
\frac{d J}{d U}=\frac{\Lambda J+\Lambda \varphi N U(1-U)-\varphi U(1-U)}{-\Lambda \varphi^{2} U(1-U)+\varphi^{-1} J+N U(1-U)},
$$

with the boundary conditions being given by Eqs. (27) and (28). Stationary points $(J=0, U=0)$ and $(J=0, U=1)$ in the phase space $(J, U)$ are node and saddle, respectively. In the following, these node and saddle points will be referred to as the leading edge (LE) and the trailing edge (TE), respectively.

If $\Lambda=\varphi^{-1}$, i.e., the TW speed $\Lambda$ is equal to the maximal propagation speed, Eqs. (22) and (23) read

$$
\begin{gathered}
-\frac{d U}{d \zeta}+\frac{d J}{d \zeta}=\varphi U(1-U) \\
-\frac{d J}{d \zeta}+\frac{d U}{d \zeta}=-\varphi^{-1} J-N U(1-U)
\end{gathered}
$$

Equations (30) and (31) are consistent with one another only if their RHSs are linked as follows:

$$
\varphi U(1-U)=\varphi^{-1} J+N U(1-U) .
$$

Therefore the flux $J$ is expressed through the concentration $U$ by the algebraic relation

$$
J=\left(\varphi^{2}-N \varphi\right) U(1-U)
$$

If $\Lambda \neq \varphi^{-1}$, then, the ODEs (25) and (26) or ODE (29) and boundary conditions given by Eqs. (27) and (28) pose a BVP. To solve it, we have to find such eigenvalues $\Lambda$ that a trajectory $J(U)$ connects singular points $(0,0)$ and $(0,1)$ in the phase space $(J, U)$. If $\Lambda=\varphi^{-1}$, then, the BVP consists of the ODE (30) supplemented with the algebraic Eq. (33) and boundary conditions given by Eqs. (27) and (28). In this case, the TW speed is known, but it is necessary to prove existence of a trajectory $J(U)$ that connects singular points $(0,0)$ and $(0,1)$ in the phase space $(J, U)$. In the particular case of vanishing advection, i.e., $N=0$, the BVP was considered by Hadeler $[35,36]$ for smooth TW solutions whose speed was lower than maximal speed, i.e., $\Lambda<\varphi^{-1}$. Recently, Bouin et al. [46] studied the same case of $N=0$, extended the analysis by Hadeler [35,36] to $\Lambda=\varphi^{-1}$, and found that (i) smooth TW solutions existed only if $\varphi<1$, (ii) TW solutions were continuous but not smooth if $\varphi=1$, and (iii) $\mathrm{TW}$ solutions were discontinuous (piecewise smooth) if $\varphi>1$. It is worth noting that formal mathematical TW solutions to the considered BVP can exist even if $\Lambda>\varphi^{-1}$, as proved at $N=0$ in [46]. However, the speed $\Lambda$ of a relevant TW solution that develops from the natural initial conditions given by Eq. (4) cannot exceed the maximal propagation speed $\varphi^{-1}$.

For other initial conditions, i.e., for initial conditions with noncompact support, asymptotic TW solution and its speed are controlled by the decay of $u(\xi, 0)$ as $\xi \rightarrow \infty$ (for a leftpropagating wave). In that case, the constraint of $\Lambda \leqslant \varphi^{-1}$ does not hold and the TW speed can exceed the maximal propagation speed, i.e., $\Lambda>\varphi^{-1}$. Such solutions are beyond the scope of the present study and we will restrict ourselves to the compact initial conditions given by Eq. (4).

In the following, the cases of $\Lambda<\varphi^{-1}$ and $\Lambda=\varphi^{-1}$ will be considered separately.

\section{A. TW speed is smaller than the maximal propagation speed, $\Lambda<\varphi^{-1}$; smooth TWs}

Solution of the nonlinear BVP given by Eqs. (25)-(28) requires consideration of the global behavior of the trajectories in the phase space. However, necessary conditions for existence of such trajectories and, in particular, a constraint that bounds the TW speed from below can be found by applying the linear analysis at the leading edge provided that the TW solution is smooth. Such a method is widely used when studying the KPP equation $[2-5,12,13,32]$.

Linearization of Eqs. (25) and (26) at the leading edge, where $U \ll 1,|J| \ll 1$, yields

$$
\begin{aligned}
\frac{d U}{d \zeta} & =\frac{\left(N-\Lambda \varphi^{2}\right) U+\varphi^{-1} J}{\Lambda^{2} \varphi^{2}-1} \\
\frac{d J}{d \zeta} & =\frac{(\Lambda \varphi N-\varphi) U+\Lambda J}{\Lambda^{2} \varphi^{2}-1}
\end{aligned}
$$


TABLE I. Summary of results obtained in Sec. III.

\begin{tabular}{llc}
\hline \hline$\varphi$ & $N$ & Result \\
\hline $0<\varphi$ & $N<\varphi-\varphi^{-1}$ & $\begin{array}{c}\text { Piecewise smooth TW with } \Lambda=\varphi^{-1} \text { and jump discontinuity } \\
\text { at the sharp leading front; see Eq. (47) } \\
0<\varphi\end{array}$ \\
& $N=\varphi-\varphi^{-1}$ & $\begin{array}{c}\text { Piecewise smooth TW with } \Lambda=\varphi^{-1} \text { and discontinuous derivatives } \\
\text { at the sharp leading front; see Eq. (51) }\end{array}$ \\
$0<\varphi<1$ & $\varphi-\varphi^{-1}<N<2$ & Smooth TW solutions with continuous spectrum $\Lambda \geqslant \Lambda_{\min }>0$ and $\Lambda_{\min } \varphi<1$ \\
$1 \leqslant \varphi$ & $\varphi-\varphi^{-1}<N<\varphi+\varphi^{-1}$ & Smooth TW solutions with continuous spectrum $\Lambda \geqslant \Lambda_{\min }>0$ and $\Lambda_{\min } \varphi<1$ \\
\hline \hline
\end{tabular}

In the vicinity of the unstable equilibrium point $(0,0)$, we can seek for the following asymptotic solution:

$$
\begin{aligned}
& U \propto \exp (-\kappa \zeta) \ll 1, \quad J \propto \exp (-\kappa \zeta) \ll 1, \\
& \kappa>0, \quad \zeta \rightarrow \infty,
\end{aligned}
$$

where $\kappa$ is the wave steepness or decay rate.

The asymptotic Eq. (36) assumes a smooth TW with infinite tail at the leading edge. At a first glance, Eq. (36) cannot be applied, because Eqs. (20) and (21) show that the concentration profile $u(\xi, \theta)$ is not perturbed ahead of the right sharp front $\xi_{r}(\theta)=\xi_{20}+\varphi^{-1} \theta$. In other words, at arbitrary $\theta>0$, there is a uniform state $u \equiv 0$ and $j \equiv 0$ if $\xi>\xi_{r}(\theta)=\xi_{20}+\varphi^{-1} \theta$; see Eq. (20). To resolve the problem, it is worth remembering that a TW is an intermediate asymptotic $[47,48]$. Accordingly, at $\theta \gg 1$, a distance between the sharp front $\xi_{r}(\theta)$ and a trajectory $\xi_{0}(\theta)$ of an arbitrary point characterized by a constant $0<u\left(\xi_{0}(\theta), \theta\right)=u_{0}<1$ grows as $\varphi^{-1} \theta-\Lambda \theta=$ $(1-\Lambda \varphi) \varphi^{-1} \theta$, because the speed of the developing TW is close to $\Lambda$ at $\theta \gg 1$. Therefore, the following inequalities $1 \ll \zeta<(1-\Lambda \varphi) \varphi^{-1} \theta$ should be satisfied in order for the use of the asymptotic Eq. (36) to be justified. In other words, a developing TW appears at a large distance from the sharp front $\xi_{r}(\theta)$.

As discussed in detail in Appendix A, the linear analysis, i.e., substitution of Eq. (36) into Eqs. (34) and (35), yields the following dispersion relation:

$$
\left(1-\Lambda^{2} \varphi^{2}\right) \kappa^{2}-\left[\Lambda\left(1-\varphi^{2}\right)+N_{B}\right] \kappa+1=0,
$$

which has two solutions:

$$
\begin{gathered}
\kappa_{+}=\frac{\Lambda\left(1-\varphi^{2}\right)+N+\sqrt{\Delta_{0}(\varphi, \Lambda, N)}}{2\left(1-\Lambda^{2} \varphi^{2}\right)}, \\
\kappa_{-}=\frac{\Lambda\left(1-\varphi^{2}\right)+N-\sqrt{\Delta_{0}(\varphi, \Lambda, N)}}{2\left(1-\Lambda^{2} \varphi^{2}\right)},
\end{gathered}
$$

where

$$
\Delta_{0}(\varphi, \Lambda, N)=\left[\Lambda\left(1-\varphi^{2}\right)+N\right]^{2}-4\left(1-\Lambda^{2} \varphi^{2}\right) .
$$

Constraint of $\Delta_{0}(\varphi, \Lambda, N) \geqslant 0$ is satisfied in particular if

$$
\Lambda_{\min +}(\varphi, N)=\frac{N\left(\varphi-\varphi^{-1}\right)+2 \sqrt{\left(\varphi+\varphi^{-1}\right)^{2}-N^{2}}}{\varphi\left(\varphi+\varphi^{-1}\right)^{2}}
$$

provided that

$$
|N| \leqslant \varphi+\varphi^{-1} .
$$

As discussed in Sec. II C, we will restrict ourselves to the case of $\Lambda=\Lambda_{\min }>0$ and, therefore, $\kappa_{+}=\kappa_{-}=\kappa_{m}$. In this case, Eqs. (38) and (39) read

$$
\kappa_{m}=\kappa_{+}=\kappa_{-}=\frac{\Lambda_{\min }\left(1-\varphi^{2}\right)+N}{2\left(1-\varphi^{2} \Lambda_{\min }^{2}\right)} .
$$

As shown in Appendix A, the constraints of $\kappa_{m}>0, \Lambda_{\min }>$ 0 , and $\Lambda_{\min } \varphi<1$ hold if $0<\varphi<1$ and $\varphi-\varphi^{-1}<N<2$ or $\varphi \geqslant 1$ and $\varphi-\varphi^{-1}<N<\varphi+\varphi^{-1}$. If $0<\varphi<1$ and $N>2$ or $\varphi \geqslant 1$ and $N>\varphi+\varphi^{-1}$, then, $\kappa_{m}>0$ and $\Lambda_{\min } \varphi<1$, but $\Lambda_{\text {min }}+$ given by Eq. (41) is negative, whereas eigenvalues of the BVP should be positive by virtue of Eq. (24). Therefore, if $N \geqslant N_{\mathrm{cr}}^{\mathrm{LA}}$, the linear analysis does not allow us to find a TW speed, i.e., pulled TWs do not exist. It is worth noting that while the linear analysis yields $N_{\mathrm{cr}}^{\mathrm{LA}}=2$ if $0<\varphi<1$ and $N_{\mathrm{cr}}^{\mathrm{LA}}=\varphi+\varphi^{-1}$ if $\varphi \geqslant 1$, transition from pulled to pushed (the speed $\Lambda$ is controlled by the interior wave shape and is larger than $\Lambda_{\text {min }}$ given by the linear analysis) TW solutions can occur even at $N_{\mathrm{cr}}<N_{\mathrm{cr}}^{\mathrm{LA}}$. Because the linear analysis does not guarantee that a phase trajectory that starts at $(J=0, U=0)$ finishes at $(J=0, U=1)$, the true critical values $N_{\text {cr }}$ cannot be determined from the linear analysis and will be found in Sec. V; see Eq. (59).

Results obtained in Appendix A hold even in a more general case, i.e., for any flux $Q$ such that $Q(u \rightarrow 0) \rightarrow$ $N u$ and for any source term $\omega(u)$ such that $\omega(u \ll 1) \leqslant u$, because linearization of this more general BVP at $u \ll 1$ results in Eqs. (A4)-(A8), with unity being substituted with $d \omega / d u(u \rightarrow 0) \leqslant 1$ in each of these equations. Furthermore,



FIG. 1. Normalized minimal speeds $\Lambda_{\min }$ for smooth TW solutions vs $\varphi$, calculated at various magnitudes $N$ of the nonlinear advection term, specified in legends. Lines show results yielded by the linear analysis at the leading edge. Circles bound intervals of relevant TW solutions, which are discussed in Sec. V. 
TABLE II. Relevant TW solutions.

\begin{tabular}{lll}
\hline \hline$N$ & $\Lambda$ & Wave type \\
\hline$N<\varphi-\varphi^{-1}$ & $\Lambda=\varphi^{-1}$ & Piecewise smooth TW with jump discontinuity at the sharp leading front; see Eq. (47) \\
$N=\varphi-\varphi^{-1}$ & $\Lambda=\varphi^{-1}$ & Piecewise smooth TW with discontinuous derivatives at the sharp leading front; see Eq. (51) \\
$\varphi-\varphi^{-1}<N<\sqrt{1+\varphi^{2}}$ & $\Lambda_{\min +}$, Eq. (41) & Smooth pulled TW solutions \\
$\sqrt{1+\varphi^{2}}<N$ & $\Lambda=N^{-1}$ & Smooth pushed TW solutions \\
\hline \hline
\end{tabular}

even if the dimensional problem parameters $\tau, \tau_{f}, D$, and $V$ vary within the TW, then, we can still arrive at Eqs. (A4)(A8) and again draw the same conclusions by normalizing the governing equations using $\tau(u \rightarrow 0), \tau_{f}(u \rightarrow 0)$, $D(u \rightarrow 0)$, and $\varphi^{2}=\tau(u \rightarrow 0) / \tau_{f}(u \rightarrow 0)$, with $N$ designating $V / \sqrt{D / \tau_{f}(u \rightarrow 0)}$ in this case. However, in order to obtain exact analytical solutions discussed in Sec. IV, the dimensional problem parameters should be independent of $u$.

Finally, it is worth noting that Eqs. (25) and (26) can also be linearized at the trailing edge. Here, behavior of these equations at $u \rightarrow 1$ is not discussed, because such a method does not yield any constraint on $\Lambda_{\text {min }}$ for any admissible $N$ or $\varphi$. This situation (a minor value of the linearized analysis at $u \rightarrow 1$ ) is typical in the theory of reaction-diffusion equations [32].

\section{B. TW speed is equal to the maximal propagation speed, $\Lambda=\varphi^{-1}$}

Let us assume that, in the case of $\Lambda=\varphi^{-1}$, a developing TW has a sharp leading front attached to the right perturbation front $\xi_{r}(\theta)$. In other words, the asymptotic TW appears directly at the right perturbation front $\xi_{r}(\theta)$. This assumption will be confirmed by numerically solving two IBVPs for hyperbolic Eqs. (10) and (11) in Sec. VI.

As already shown, the case of $\Lambda=\varphi^{-1}$ is described by Eq. (30) supplemented with Eq. (33). Substitution of Eq. (33) into Eq. (30) yields the following first-order nonlinear ODE:

$$
a(U, \varphi, N) \frac{d U}{d \zeta}=-\varphi U(1-U)
$$

where

$$
a(U, \varphi, N)=-m+2(m+1) U, \quad m=\varphi^{2}-1-N \varphi .
$$

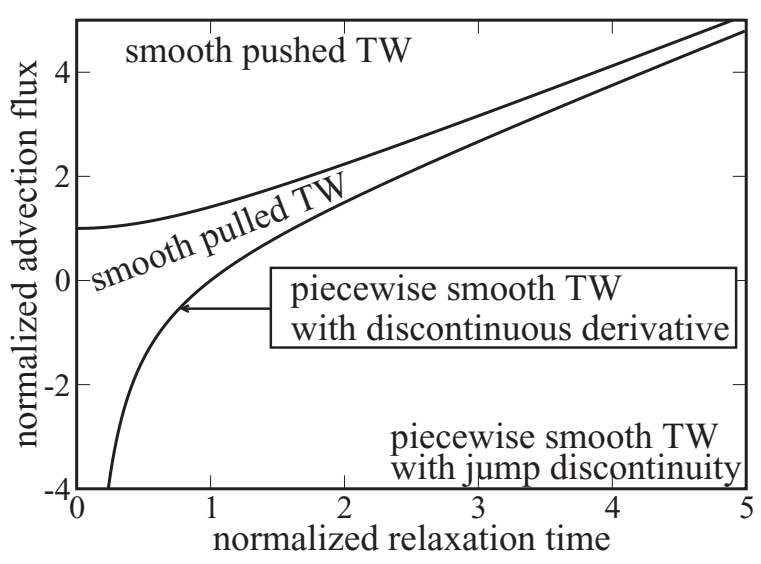

FIG. 2. Various types of relevant TW solutions.
Contrary to Eqs. (25) and (26), an implicit solution to Eq. (44) can be obtained analytically; see Appendix B. Qualitative behavior of the solution depends on the sign of the coefficient $a(U, \varphi, N)$ defined by Eq. (38). An analysis of Eq. (45) shows five different cases:

(i) if $N<\varphi-\varphi^{-1}$, then, $m>0, a\left(U_{*}<U \leqslant 1 ; \varphi, N\right)>$ $0, a\left(U_{*} ; \varphi, N\right)=0$, and $a\left(0 \leqslant U<U_{*} ; \varphi, N\right)<0$;

(ii) if $N=\varphi-\varphi^{-1}$, then, $m=0, a(U ; \varphi, N)=2 U \geqslant 0$;

(iii) if $\varphi-\varphi^{-1}<N<\varphi+\varphi^{-1}$, then, $-2<m<0$, $a(U ; \varphi, N)>0$;

(iv) if $N=\varphi+\varphi^{-1}$, then, $m=-2, \quad a(U ; \varphi, N)=$ $2(1-U) \geqslant 0$;

(v) if $N>\varphi+\varphi^{-1}$, then, $m<-2, a\left(U_{*}<U \leqslant 1\right.$; $\varphi, N)<0, a\left(U_{*} ; \varphi, N\right)=0$, and $a\left(0 \leqslant U<U_{*} ; \varphi, N\right)>0$.

Here,

$$
\begin{aligned}
U_{*} & =\frac{m}{2(m+1)}, \quad 0<U_{*}<\frac{1}{2} \quad \text { in case }(\mathrm{i}), \\
\frac{1}{2} & <U_{*}<1 \text { in case }(\mathrm{v}) .
\end{aligned}
$$

It is worth remembering that the derivative $d U / d \zeta<0$ should be negative within a TW that propagates from left to right. Therefore the coefficient $a(U ; \varphi, N)$ should be positive, i.e., $a(U ; \varphi, N)>0$. This constraint holds in the entire interval of $-\infty<\zeta<\infty$ solely in case (iii). All five cases are analyzed in Appendix B. Cases (iii)-(v) have no physical interest, because the constraint given by Eq. (20) does not hold in these three cases and these TW solutions have infinitely long tails at their leading edges.

In case (i) of $N<\varphi-\varphi^{-1}$, the TW solution

$$
\begin{aligned}
U^{m}(1-U)^{n} & =U_{L}^{m}\left(1-U_{L}\right)^{n} \exp \left[\varphi\left(\zeta-\zeta_{0}\right)\right], \quad \text { if } \zeta<\zeta_{0}, \\
U & =0, \quad \text { if } \zeta>\zeta_{0},
\end{aligned}
$$

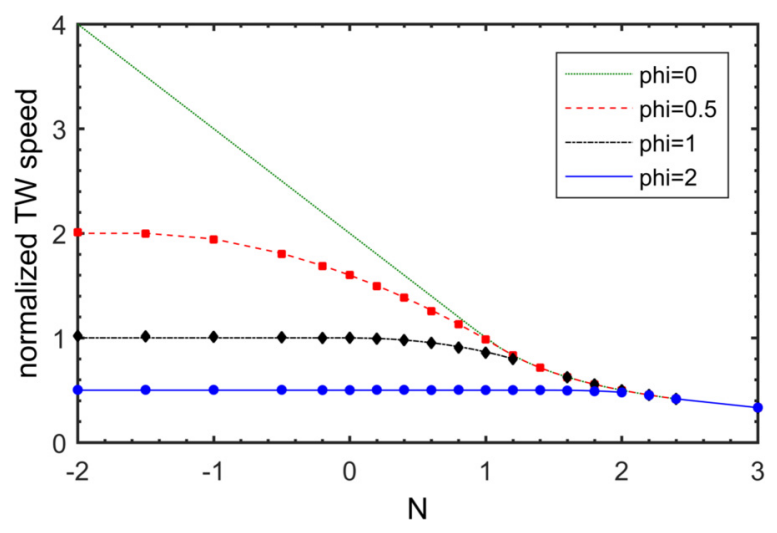

FIG. 3. TW speeds. Lines and symbols show analytical and numerical results, respectively. 
(a)

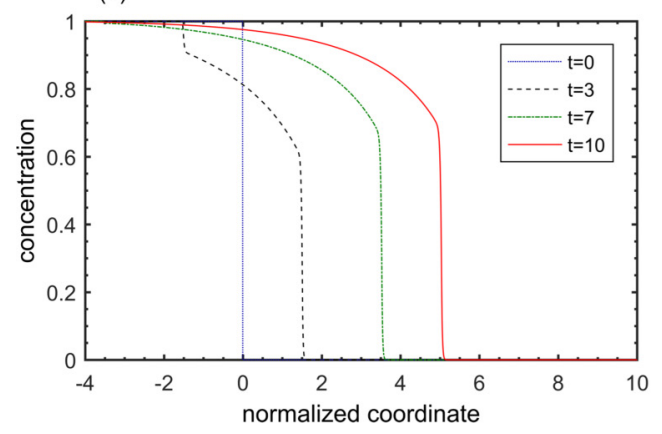

(b)

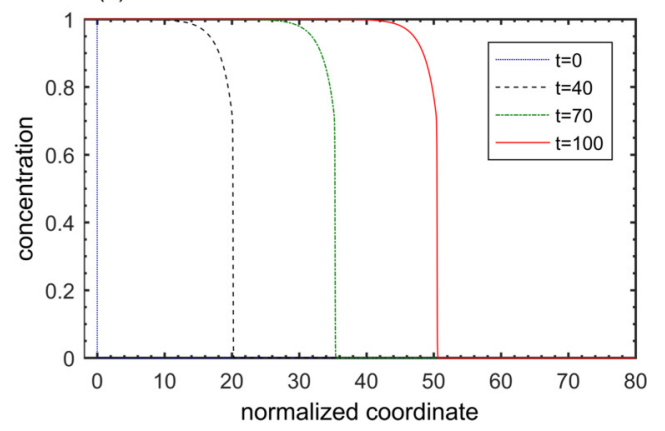

FIG. 4. Wave evolution from the step initial conditions, see Eq. (60), computed at $\varphi=2$ and $N=0.4$. (a) Short-term evolution. (b) Long-term evolution.

found in Appendix B 1, is piecewise smooth and has jump discontinuity at the sharp leading front $\zeta=\zeta_{0}$ from $U_{L}=$ $U\left(\zeta_{0}-\right)$ and $J_{L}=J\left(\zeta_{0}-\right)$ just behind (to the left) of the jump to $U_{R}=U\left(\zeta_{0}+\right)$ and $J_{R}=J\left(\zeta_{0}+\right)$, respectively, just ahead (to the right) of the jump. Here,

$$
\begin{gathered}
U_{L}=2 U_{*}=\frac{m}{m+1}<1, \quad 0<U_{L}<1, \\
m=\varphi^{2}-1-N \varphi>0, \quad n=m+2,
\end{gathered}
$$

as shown in Appendix B 1. Substitution of $U_{L}$ given by Eq. (48) into Eq. (33) yields the following magnitude of the flux jump:

$$
J_{L}=U_{L},
$$

i.e., the jump in the flux $J$ is equal to the jump in the concentration $U$.

It is worth noting that $U_{L}=0$ if $N=\varphi-\varphi^{-1}$ and $U_{L} \rightarrow 1$ if $N \rightarrow-\infty$ at a constant $\varphi$, while the case of $N \rightarrow \infty$ is not considered, because $N<\varphi-\varphi^{-1}$, as stated in the beginning of the present subsection.

This solution approaches the trailing edge as follows

$$
U \rightarrow 1-U_{L}^{m / n}\left(1-U_{L}\right) \exp \left[\left(\varphi+\varphi^{-1}-N\right)^{-1} \zeta\right],
$$

$\zeta \rightarrow-\infty$.

The length of the trailing tail of the TW increases with decreasing $\varphi$. If $\varphi \rightarrow 0$ and $N$ is appropriately varied in order for the constraint of $N<\varphi-\varphi^{-1}$ to hold, then, the TW speed given by Eq. (24) tends to infinity, in line with the case of $\Lambda \varphi=1$.

In the case of $N=0$ (advection is absent), the piecewise smooth solutions were recently found by Bouin et al. [46].

In case (ii) of $N=\varphi-\varphi^{-1}$ and $a(U ; \varphi, N)=2 U$, the TW solution

$$
U(\zeta)=\left\{\begin{array}{ll}
1-\exp \left(\frac{\varphi}{2} \zeta\right) & \text { if } \zeta \leqslant 0 \\
0 & \text { if } \zeta>0
\end{array},\right.
$$

found in Appendix B 2, is piecewise smooth TW and has discontinuous derivatives at the sharp leading front, i.e., at $\zeta=0$. Equation (51) shows that the length of the TW tail increases with decreasing $\varphi$, thus, allowing Eq. (24) to yield $\Lambda \rightarrow \infty$ at $\varphi \rightarrow 0$, in line with considered case of $\Lambda \varphi=1$.

Substitution of $N=\varphi-\varphi^{-1}$ into Eq. (33) yields the following positive flux:

$$
J=U(1-U)>0
$$

and the product $J d U / d \zeta<0$ as for gradient diffusion.

A summary of results obtained by linearizing the studied BVP at the leading edge is provided in Table I and in Fig. 1(a). For any finite relaxation time $(\varphi>0)$, there are piecewise smooth TW solutions, provided that the magnitude $N$ of the nonlinear advection term does not exceed a critical value of $\varphi-\varphi^{-1}$, which may be negative.

If the relaxation time is short $(0<\varphi<1)$, the linear analysis yields a minimal propagation speed provided that the magnitude of the nonlinear advection term is moderate (a)



(b)

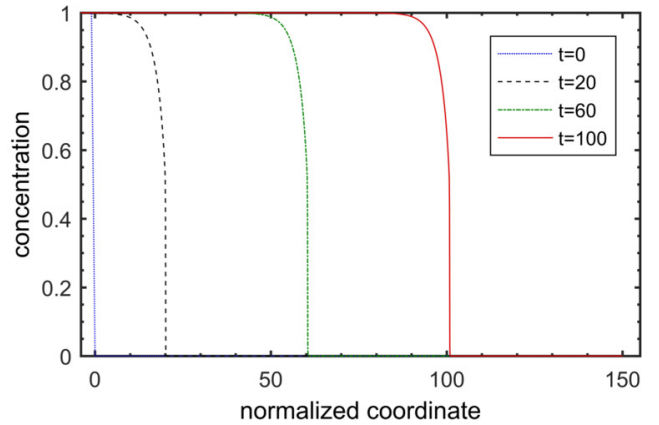

FIG. 5. Wave evolution from the ramp initial conditions, see Eq. (62), computed at $\varphi=1$ and $N=-1$. (a) Short-term evolution. (b) Long-term evolution. 
(a)



(b)

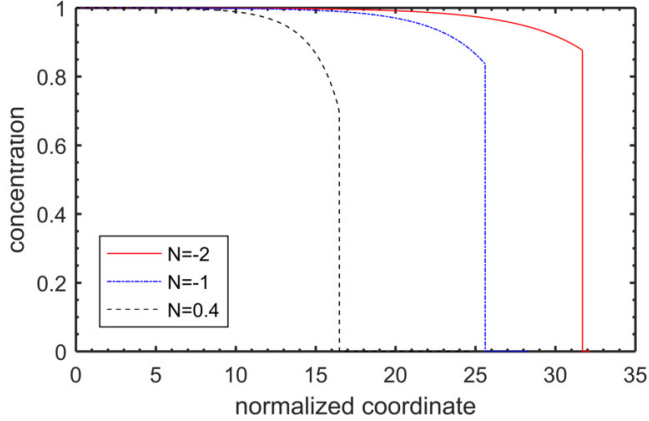

FIG. 6. Piecewise smooth discontinuous TW solutions obtained numerically at various $N<\varphi-\varphi^{-1}$. (a) $\varphi=0.5$ and (b) $\varphi=2$. In all cases, $U(0)=0.9999$.

( $\varphi-\varphi^{-1}<N<2$ ), with negative values of $N$ being again admissible. If the relaxation time is long $(\varphi \geqslant 1)$, the advection-flux magnitude should be within an interval of $\varphi-\varphi^{-1}<N<\varphi+\varphi^{-1}$ in order for the linear analysis to yield $\Lambda_{\min }>0$. For negative and moderately large positive $N$, the minimal smooth TW speed $\Lambda_{\text {min }}$ is decreased when the relaxation time is increased; see Fig. 1(a) [Fig. 1(b) will be discussed in Sec. V]. If $N$ is sufficiently large, the dependence of $\Lambda_{\min }(\varphi)$ is nonmonotonous and there is a local peak of $\Lambda_{\min }(\varphi)$, e.g., see brown dotted line in Fig. 1(a).

\section{AN EXPLICIT SMOOTH PUSHED TW SOLUTION}

As noted in Sec. II C, the mathematical theory and various methods of finding pushed TW solutions to parabolic reactiondiffusion PDE (21) were developed for a long time, as reviewed elsewhere [12,32]. In particular, pushed TW solutions to various subclasses of Eq. (21) are known, with their decay rates belonging to the $\kappa_{+}$branch given by Eq. (38) with $\varphi=0$. In other words, for such pushed TW solutions, the spectrum $\Lambda(\kappa)$ consists only of an isolated discrete point $\Lambda=\Lambda_{p}\left(\kappa_{+}\right)$. In the present section, a more general case of $\varphi>0$ is considered and an explicit pushed TW solution to Eqs. (22) and (23) is found.

The solution is as follows:

$$
U=\frac{1}{\exp (N \zeta)+1}, \quad J=0, \quad-\infty<\zeta<\infty
$$

(a)

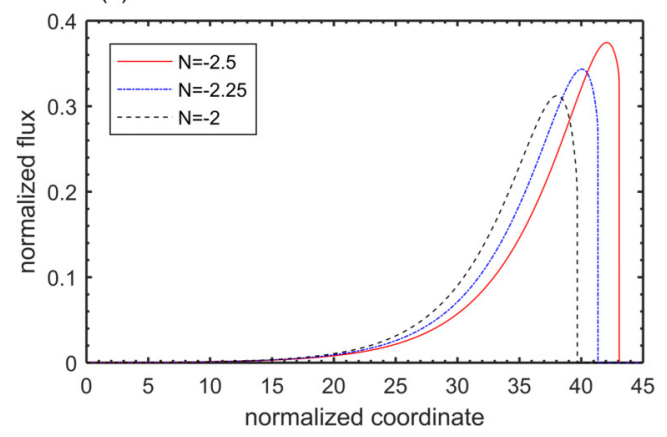

and is smooth in the entire interval of $-\infty<\zeta<\infty$. Here, we have set $U(0)=1 / 2$ without loss of generality, because Eqs. (22) and (23) are invariant with respect to space shifts.

One can easily check that (i) Eqs. (22) and (23) read

$$
\begin{aligned}
& -\Lambda \frac{d U}{d \zeta}=U(1-U), \\
& \frac{d U}{d \zeta}=-N U(1-U)
\end{aligned}
$$

in the case of $J=0$, (ii) Eqs. (54) and (55) are consistent with one another if the speed of the TW is given by

$$
\Lambda=N^{-1} \text {; }
$$

and (iii) Eq. (53) satisfies both Eqs. (54) and (55) if Eq. (56) holds. The decay rate of the considered TW solution is equal to

$$
\kappa=N
$$

Thus, for this explicit solution the TW speed is decreased when the magnitude of the advection term is increased (e.g., turbulent flame speed is reduced due to countergradient scalar transport), but the normalized decay rate is equal to this normalized magnitude.

Because the TW is smooth, its speed $\Lambda$ and decay rate $\kappa$, given by Eqs. (56) and (57), respectively, should satisfy the dispersion relation (37). The wave speed given by Eq. (56) should be higher than $\Lambda_{\min }$ given by Eq. (41), but lower than

(b)

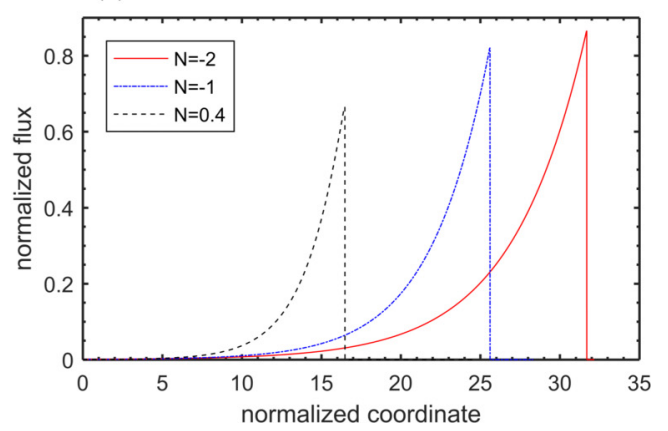

FIG. 7. Normalized fluxes associated with the piecewise smooth discontinuous TW solutions obtained numerically at various $N<\varphi-\varphi^{-1}$. (a) $\varphi=0.5$ and (b) $\varphi=2$. In all cases, $U(0)=0.9999$. 

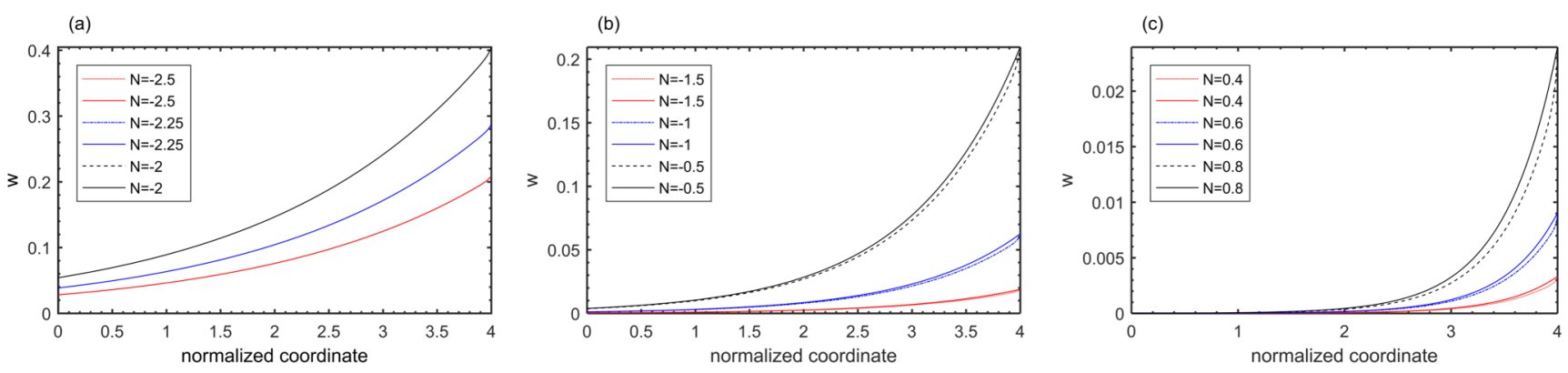

FIG. 8. Spatial profiles of $w=U_{L}^{m}\left(1-U_{L}\right)^{n} \exp \left[\varphi\left(\zeta-\zeta_{0}\right)\right]$ and $w_{\text {num }}=u_{\text {num }}^{m}\left(1-u_{\text {num }}\right)^{n}$ obtained at various $N<\varphi-\varphi^{-1}$. (a) $\varphi=0.5$, (b) $\varphi=1$, and (c) $\varphi=2$. In all cases, $\zeta_{0}=4$. Solid and dashed lines show numerical and analytical solutions, respectively.

the maximal propagation speed, i.e., $\Lambda<\varphi^{-1}$. Therefore, the pushed TW solution can only be relevant if

$$
N>\varphi \text {. }
$$

Substituting $\Lambda=N^{-1}$ into Eq. (38), one can easily check that the decay rate $\kappa=N$ given by Eq. (57) belongs to the $\kappa_{+}$branch of the dispersion relation (37). Accordingly, via an analogy with previous theoretical results summarized in Sec. II C, we can classify the found TW solution given by Eqs. (53), (56), and (57) to be the pushed TW. It is worth noting that this TW solution does not depend on $\varphi$ and is associated with vanishing flux $J=0$. Thus, the relaxation affects neither the speed nor the structure of the pushed TW solutions. In the particular case of $\varphi=0$ (lack of memory), the pushed TW solutions given by the same Eqs. (53), (56), and (57) were reported in [19-21].

\section{SELECTION OF RELEVANT TW SOLUTIONS}

The above analysis shows that the studied BVP does not have a unique solution. There are three classes of TW solutions. First, the linearized BVP admits a family of TW solutions with a continuous spectrum of eigenvalues $\Lambda_{\min } \leqslant \Lambda<\varphi^{-1}$, with the solutions being smooth in the entire interval of $-\infty<\zeta<\infty$. Second, at $N>\varphi$, there is an explicit pushed TW solution to the nonlinear BVP, with the solution being also smooth in the entire interval of $-\infty<\zeta<\infty$. Third, there are two piecewise smooth TW solutions that propagate at the maximal relevant speed $\Lambda=\varphi^{-1}$ : (i) continuous TW solution with discontinuous derivatives at the sharp leading front, and (ii) discontinuous TW solution with a jump at the sharp leading front. According to Table I and Eq. (49), domains of $(\varphi, N)$ for (i) the smooth pulled and pushed TWs and (ii) the piecewise smooth TWs are not overlapped. Therefore, a problem of selection between the smooth pulled or pushed TWs and the piecewise smooth TWs does not arise.

To the contrary, the smooth pulled and pushed TW solutions have common subdomains in two cases: (i) $\varphi<N<2$ if $\varphi<$ 1 , and (ii) $\varphi \geqslant 1$. Accordingly, a selection problem should be solved in these two cases. For this purpose, we will invoke a principle of the maximal decay rate at the leading edge $[12,43-45]$ and will compare the decay rates $\kappa$ of the smooth TW solutions in order to find the speed of the relevant solution as a function of $N$ at a constant $\varphi$.

In order to compare the decay rates given by Eqs. (43) and (57), let us, first, find a value of $N=N_{\mathrm{cr}}$ at which the two decay rates are equal to one another at the same TW speed. Substituting $\Lambda_{\min +}=\Lambda=N^{-1}$ and $\kappa_{m}=\kappa_{+}=N$ into Eqs. (43), we obtain

$$
N_{\mathrm{cr}}=\sqrt{1+\varphi^{2}}>\varphi .
$$

Second, by differentiating Eq. (41) with respect to $N$, one can show that the lowest speed $\Lambda_{\text {min }}$ and, hence, the decay rate $\kappa_{m}$ given by Eq. (43) are monotonously decreasing functions of $N$ in the range of $\varphi-\varphi^{-1}<N$, provided that Eq. (42) holds. To the contrary, the decay rate $\kappa_{p}$ of the pushed solution is increased by $N$; see Eq. (57). Hence, if $\kappa_{m}\left(N=N_{\mathrm{cr}}\right)=\kappa_{p}=N$, then, $\kappa_{m}\left(N>N_{\mathrm{cr}}\right)<N_{\mathrm{cr}}<N=$ $\kappa_{p}$ and the push TW is selected at $N>N_{\text {cr }}=\sqrt{1+\varphi^{2}}$.

Relevant TW speeds resulting from the above analysis are summarized in Table II, with the minimal speeds $\Lambda_{\text {min }+}$ of relevant pulled smooth TW solutions being plotted in Fig. 1(b). When compared to results obtained earlier [19-21] in the case of infinitely short relaxation time $(\varphi=0)$, the following three major effects of the relaxation time are worth emphasizing.

First, if $N \leqslant \varphi-\varphi^{-1}$, then, TW solutions are piecewise smooth. An increase in the magnitude $N$ of the nonlinear advection flux can make relevant TW solutions smooth in the entire spatial domain. In particular, if $\varphi^{2}>1$ (slow relaxation), then $N$ should be sufficiently large in order for TW solution to be smooth in the entire spatial domain. For instance, in the case of a slow relaxation, countergradient transport in premixed turbulent flames serves to make the spatial profile of

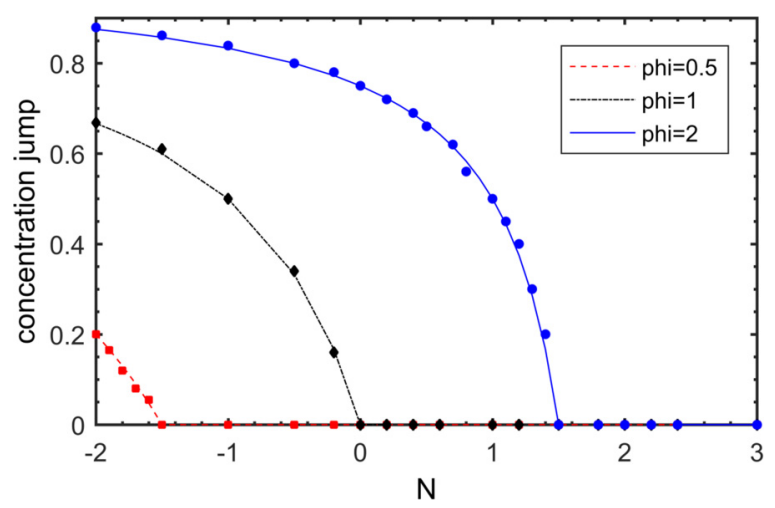

FIG. 9. Jump magnitudes for piecewise discontinuous TW solutions. Lines were calculated using Eq. (48), whereas symbols were numerically obtained. 
(a)

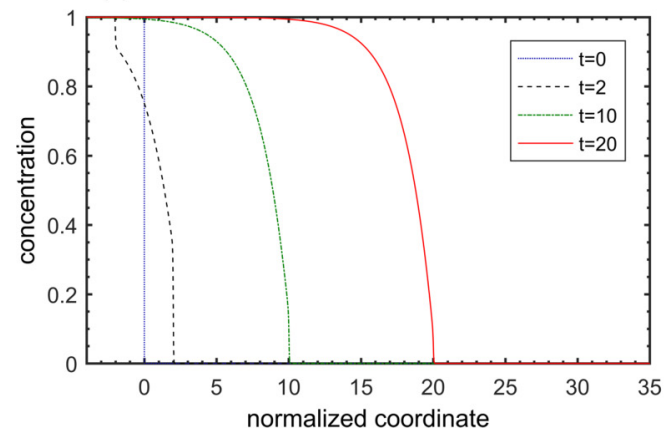

(b)

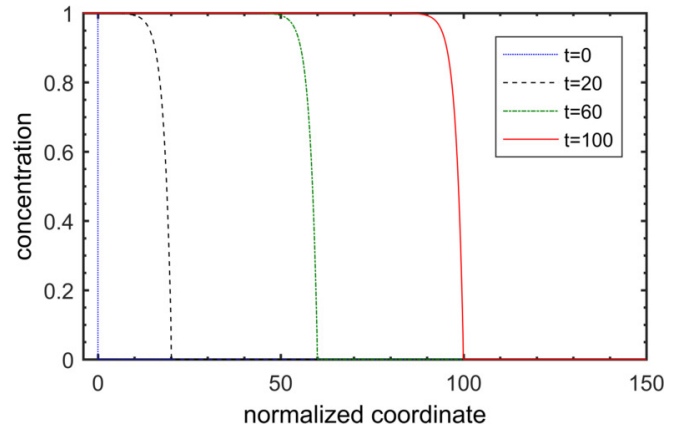

FIG. 10. Wave evolution from the step initial conditions, see Eq. (60), computed at $\varphi=1$ and $N=\varphi-\varphi^{-1}=0$. (a) Short-term evolution. (b) Long-term evolution.

the mean combustion progress variable smooth within a TW flame brush.

Second, while the speed of a relevant pushed TW solution is independent of the relaxation time, the minimal speed $\Lambda_{\min }+$ of a relevant pulled TW solution is decreased when the relaxation time is increased; see Fig. 1(b). Therefore, the memory effects can, e.g., reduce turbulent flame speed provided that the flame is pulled, i.e., $N$ is sufficiently low.

Third, an increase in the relaxation time results in increasing the critical value $N_{\mathrm{cr}}=\sqrt{1+\varphi^{2}}$ of the magnitude of the nonlinear advection term, associated with the transition from pulled to pushed relevant TWs. In other words, an increase in the relaxation time can cause a transition from pushed to pulled relevant TWs. Accordingly, while the relaxation time affects neither the speed nor the structure of pushed relevant TWs, the relaxation time does affect the critical conditions under which the pushed TWs become relevant.

Finally, various types of relevant TW solutions are summarized in Fig. 2.

\section{NUMERICAL SIMULATION}

\section{A. Formulation of IBVP}

Because the analytical results obtained in the previous section are strongly based on the steepness criterion [12,43-45], which is widely accepted, but has not yet been proved rigorously, the above analysis requires validation in numerical simulations. In particular, it should be demonstrated that

(a)

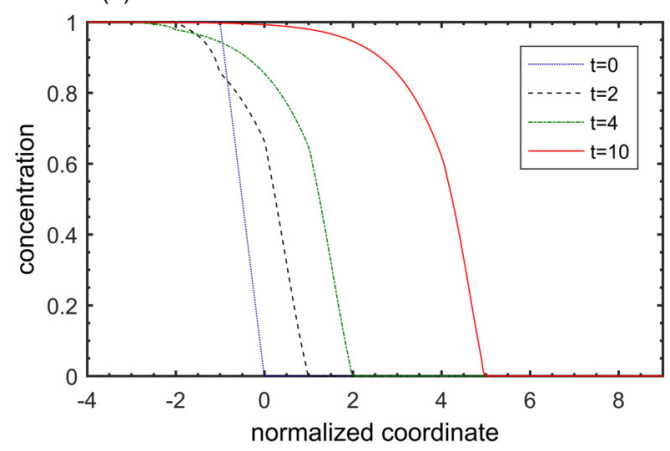

solutions to the IBVP given by Eqs. (10) and (11) with $Q=u(1-u)$ and $\omega=u(1-u)$ and initial and boundary conditions stated by Eqs. (4) and (6), respectively, tend to the TW solutions obtained in the previous sections. Numerical simulations are also required to support the piecewise smooth TW solutions that propagate at the maximal speed $\Lambda=\varphi^{-1}$, see Sec. III B, and to study evolution of transient solutions to such TW solutions.

In numerical simulations, it is convenient to deal with Eqs. (13)-(15) where $Q=u(1-u)$ and $\omega=u(1-u)$. In the present work, numerical simulations were carried out for two types of initial conditions for functions $u_{+}$and $u_{-}$: (i) discontinuous step functions,

$$
\begin{gathered}
u_{+}(\xi, \theta=0)=u_{-}(\xi, \theta=0)= \begin{cases}1 / 2, & \xi \leqslant 0 \\
0, & \xi>0\end{cases} \\
u(\xi, \theta=0)=H(-\xi), \quad \hat{j}(\xi, \theta=0)=0,
\end{gathered}
$$

and (ii) continuous, but not smooth, ramp functions,

$$
u_{+}(\xi, \theta=0)=u_{-}(\xi, \theta=0)=\left\{\begin{array}{lr}
1 / 2, & \xi \leqslant-1 \\
(1-\xi) / 2, & -1 \leqslant \xi \leqslant 0, \\
0, & \xi>0
\end{array}\right.
$$

$$
u(\xi, \theta=0)=\left\{\begin{array}{lc}
1, & \xi \leqslant-1 \\
1-\xi, & -1 \leqslant \xi \leqslant 0, \hat{j}(\xi, \theta=0)=0 \\
0, & \xi>0
\end{array}\right.
$$

(b)

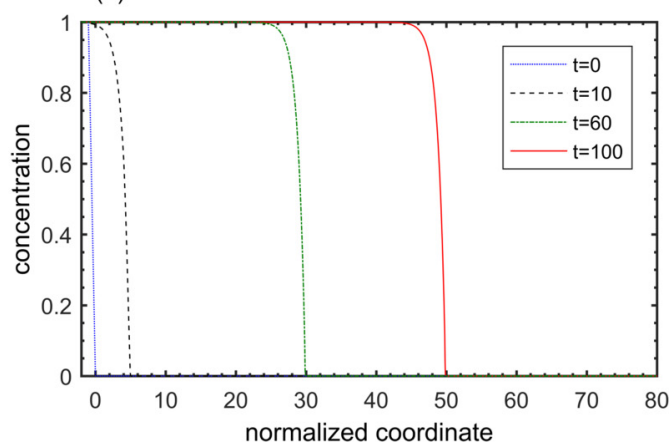

FIG. 11. Wave evolution from the ramp initial conditions, see Eq. (62), computed at $\varphi=2$ and $N=\varphi-\varphi^{-1}=1.5$. (a) Short-term evolution. (b) Long-term evolution. 


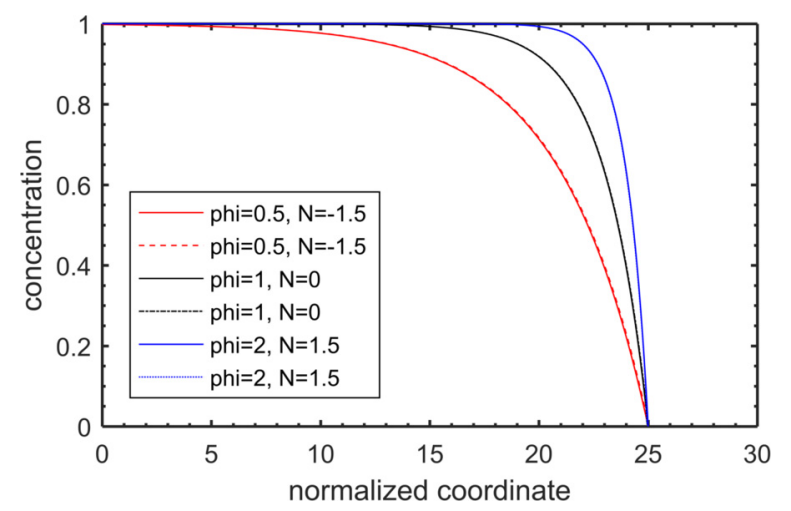

FIG. 12. Piecewise smooth continuous TW solutions obtained numerically at various $\varphi$ and $N=\varphi-\varphi^{-1}$. In all cases, $U(25)=0$. Numerical and analytical results are shown in solid and dashed lines, respectively.

with initial jumps in the derivatives $\partial u_{+}(\xi) / \partial \xi, \partial u_{-}(\xi) / \partial \xi$, and $\partial u(\xi) / \partial \xi$.

Boundary conditions read

$u_{+}(-\infty, \theta)=u_{-}(-\infty, \theta)=1 / 2, u_{+}(\infty, \theta)=u_{-}(\infty, \theta)=0$.

The numerical scheme and details of the calculations are discussed in Appendix C.

\section{B. Numerical results}

\section{TWs speeds}

The asymptotic TW speeds $\Lambda$ obtained analytically and numerically for various $N$ and $\varphi=0.5, \varphi=1$, or $\varphi=2$ are compared in Fig. 3, with the same $\Lambda$ and TW solutions being computed using two different initial conditions; see Eqs. (60) and (62). The theoretical results are summarized in Table II. The pulled TW solutions exist in the domain of $\varphi-\varphi^{-1}<$ $N<\sqrt{1+\varphi^{2}}$, with their speed $\Lambda_{\min +}<\varphi^{-1}$ being given by Eq. (41). The pushed TW solutions exist in the domain of $N>\sqrt{1+\varphi^{2}}$, with their speed being equal to $\Lambda=N^{-1}$. The transition from the pulled to pushed TW solutions occurs at $N=N_{\text {cr }}=\sqrt{1+\varphi^{2}}$. The piecewise smooth TW solutions with discontinuous derivatives at the sharp leading front exist at $N=\varphi-\varphi^{-1}$, with their speed being equal to $\Lambda=\varphi^{-1}$. The piecewise smooth TW solutions with jump discontinuity at

(a)

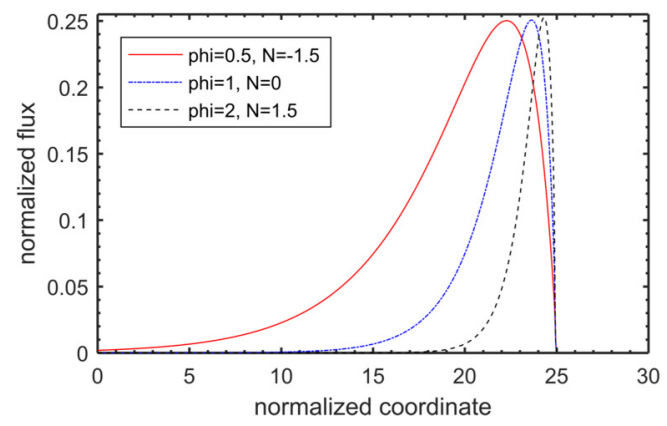

the sharp leading front exist at $N<\varphi-\varphi^{-1}$, with their speed being equal to $\Lambda=\varphi^{-1}$. In the case of $\varphi=0$, the theoretical results reduce to

$$
\Lambda=\left\{\begin{array}{ll}
2-N & \text { if }-\infty<N<1 \\
\frac{1}{N} & \text { if } N>1
\end{array} .\right.
$$

While this expression was analytically obtained and numerically validated earlier [19], it is also shown in Fig. 3 for completeness.

In all studied cases, agreement between the analytical and numerical results is very good, thus validating the principle of the maximal decay rate, which was invoked for selection of relevant smooth TW solutions.

While Fig. 3 is the major output of the present simulations, more numerical results will be reported in the rest of the paper in order (i) to address evolution of transient waves to TWs, (ii) to demonstrate appearance of piecewise smooth TW solutions, (iii) to gain an insight into features of the TW solutions, and (iv) to validate both analytical and numerical results by showing agreement between them.

\section{2. $N<\varphi-\varphi^{-1}$ : Piecewise smooth discontinuous TWs}

Numerical results obtained in the cases of $\{\varphi=2, N=0.4\}$ and $\{\varphi=1, N=-1\}$ are reported in Figs. 4 and 5, respectively. For illustration purposes, Figs. 4 and 5 show results computed using initial conditions stated by Eqs. (60) and (62), respectively. It is worth stressing that, in all simulated cases, the asymptotic numerical TW solutions obtained using initial conditions given by Eqs. (60) or (62) were indistinguishable, in line with the theoretical analysis.

If Eq. (60) is used, see Fig. 4, then the initial unit jump propagates with the right sharp front at a speed equal to $\varphi^{-1}$, in line with Eq. (19). The jump amplitude decays with time and tends to an asymptotic value, which depends on $\varphi$ and $N$, in line with Eq. (48), as will be shown later. The asymptotic TW solution is attached to the right sharp front behind the jump, i.e., the TW propagates at the speed $\varphi^{-1}$ of the right sharp front, as argued in Sec. III B.

For the ramp initial conditions given by Eq. (62), see Fig. 5, the derivative $\partial u / \partial \xi$ is discontinuous, but the initial concentration jump is absent. Nevertheless, Fig. 5 shows the appearance and subsequent growth of the concentration jump.

Figures 6 and 7 show piecewise smooth discontinuous TW solutions for concentration and fluxes, respectively, obtained

(b)



FIG. 13. Normalized flux associated with the piecewise smooth continuous TW solutions obtained numerically at various $\varphi$ and $N=$ $\varphi-\varphi^{-1}$. (a) Fluxes vs normalized coordinate graph, in all cases, $U(25)=0$. (b) Fluxes vs concentration graph. 

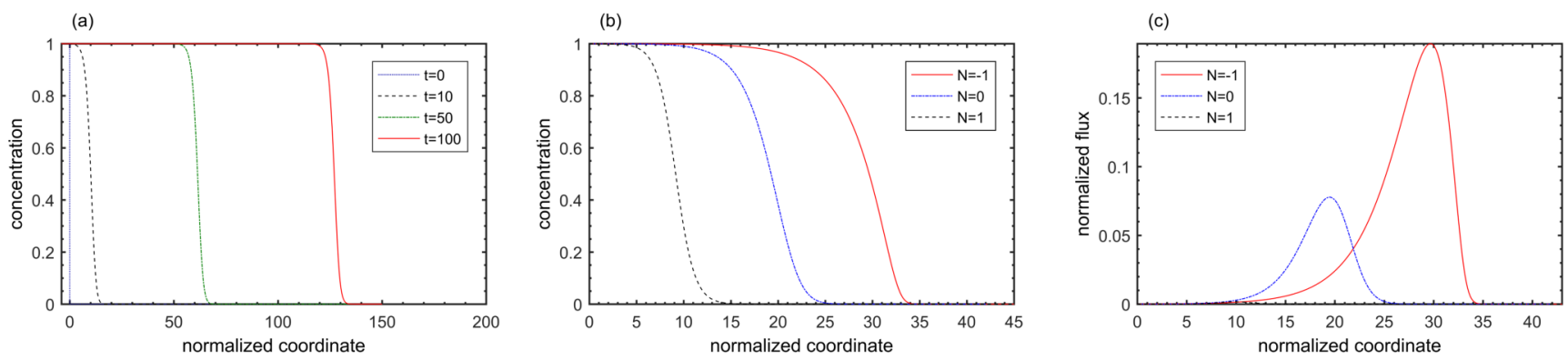

FIG. 14. (a) Wave evolution from the step initial conditions given by Eq. (60), $N=0.5$. (b) Smooth pulled TW solutions, in all cases $U(0)=0.9999$. (c) Spatial profiles of normalized flux. $\varphi=0.5$ for (a), (b) and (c).

numerically at $\varphi=0.5$ and $\varphi=2$ and $N<\varphi-\varphi^{-1}$. If $\varphi$ is kept constant and $N$ is decreased, then the concentration jump amplitude is increased, in line with Eq. (48). The length of the trailing tail increases (i) with decreasing $\varphi$ at a constant $N$, cf. cases with $N=-2$, and (ii) with decreasing $N$ at a constant fixed $\varphi$.

Precision of the obtained numerical solutions is demonstrated in Fig. 8, where functions $w=U_{L}^{m}\left(1-U_{L}\right)^{n}$ $\exp \left[\varphi\left(\zeta-\zeta_{0}\right)\right]$ and $w_{\text {num }}=u_{\text {num }}^{m}\left(1-u_{\text {num }}\right)^{n}$ are compared, because the analytical solution given by Eq. (47) is implicit. Here, $\zeta_{0}$ is associated with the leading front, where a jump from $U=U_{L}$ to $U=0$ occurs, and $u_{\text {num }}$ is a numerical solution to PDEs (13) and (14) with $\omega=Q=u(1-u)$. In all cases solid (numerical data) and dashed (analytical results) lines are close to one another or even indistinguishable, e.g., at $\varphi=0.5$.

Figure 9 shows that the jump magnitudes $U_{L}$ evaluated analytically (lines), see Eq. (48), and numerically (symbols) agree very well at various $\varphi$ and $N<\varphi-\varphi^{-1}$.

\section{3. $N=\varphi-\varphi^{-1}$ : Piecewise smooth continuous $T W s$}

Numerical results obtained using the step initial conditions given by Eq. (60) in the case of $\varphi=1, N=\varphi-\varphi^{-1}=0$ and the ramp initial conditions given by Eq. (62) in the case of $\varphi=2, N=\varphi-\varphi^{-1}=1.5$ are reported in Figs. 10 and 11 , respectively.

If Eq. (60) is used, see Fig. 10, then, the initial unit jump propagates at a speed equal to $\varphi^{-1}$, in line with Eq. (19). The jump amplitude decays with time and asymptotically vanishes. The derivative $\partial u / \partial \xi$ is unbounded at the wave sharp front at the initial instant. Subsequently, the derivative magnitude decays with time and tends to $-\varphi / 2$ at the wave sharp front, in line with Eq. (51). The developing wave is permanently attached to the right sharp front behind the jump, i.e., both the developing wave and the TW propagate at the speed $\varphi^{-1}$ of the right sharp front, as argued in Sec. III B.

For the ramp initial conditions given by Eq. (62), the initial concentration jump is absent, but the derivative $\partial u / \partial \xi$ is discontinuous and is equal to -1 at the ramp. Figure 11 shows the wave evolution with time and confirms that the normalized derivative at the front approaches $-\varphi / 2$, in line with Eq. (51).

Figure 12 compares piecewise smooth discontinuous TW solutions that were obtained analytically using Eq. (51), see solid lines, and numerically, see dashed lines, at various $\varphi$ and $N=\varphi-\varphi^{-1}$. The numerical and analytical results are essentially indistinguishable. The length of the trailing tail increases with decreasing $\varphi$.

Figure 13 shows profiles of the normalized flux $J$ obtained numerically in the same three cases, i.e., $\varphi=0.5(N=-1.5)$, $\varphi=1(N=0)$, and $\varphi=2(N=1.5)$. In line with Eq. (52), the numerical profiles of $J$ vs $U$, see Fig. 13(b), are very similar to the logistic expression.

$$
\text { 4. } \varphi-\varphi^{-1}<N<\sqrt{1+\varphi^{2}} \text { : Pulled TWs }
$$

Typical transient numerical results obtained at $\varphi-\varphi^{-1}<$ $N<\sqrt{1+\varphi^{2}}$ using the step initial conditions given by Eq. (60) are reported in Fig. 14(a). Figure 14(b) shows typical pulled TW solutions. Typical spatial profiles $J(\xi)$ of the normalized flux are plotted in Fig. 14(c). Note that the dashed (a)

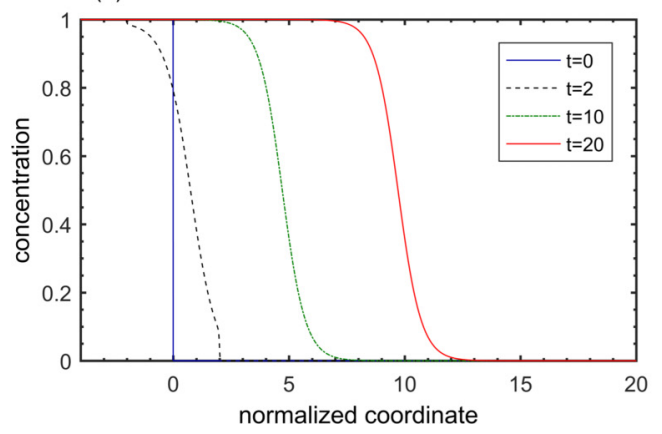

(b)

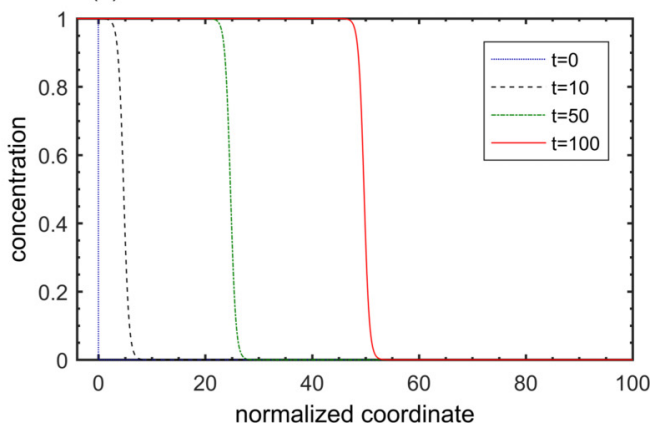

FIG. 15. Wave evolution from the step initial conditions, see Eq. (60), computed at $\varphi=1$ and $N=2$. (a) Short-term evolution. (b) Long-term evolution. 

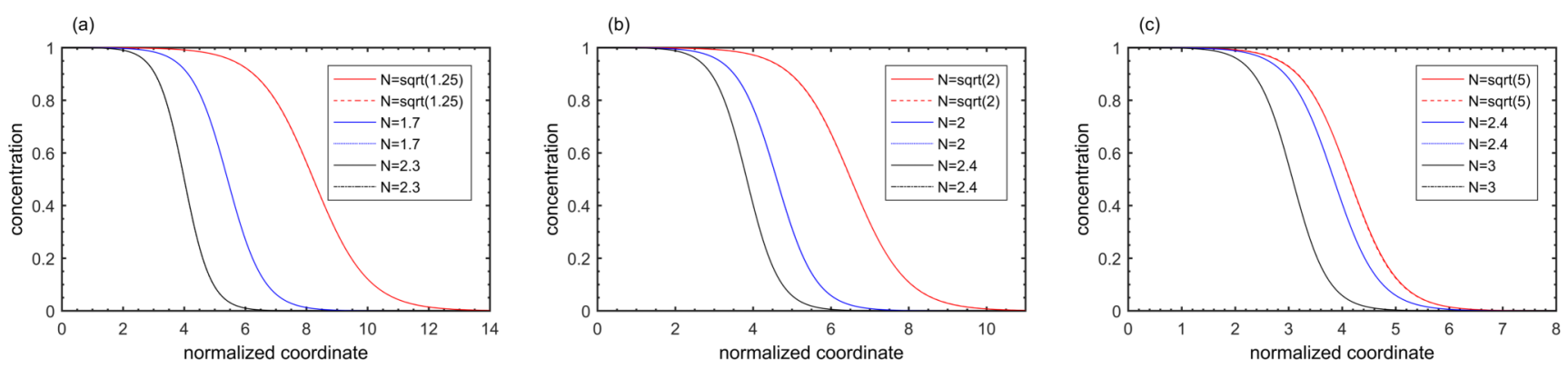

FIG. 16. Analytical (dashed lines) and numerical (solid lines) pushed TW solutions obtained at various $N>\sqrt{1+\varphi^{2}}$. (a) $\varphi=0.5$, (b) $\varphi=1$, and (c) $\varphi=2$. In all cases $U(0)=0.9999$.

line in Fig. 14(c) is very close to the ordinate axis, i.e., the flux magnitude is strongly reduced when $N$ is increased.

\section{5. $N>\sqrt{1+\varphi^{2}}:$ Pushed TWs}

Typical transient numerical results obtained at $N>$ $\sqrt{1+\varphi^{2}}$ using the step initial conditions given by Eq. (60) are reported in Fig. 15. Figure 16 validates both the present numerical simulations and the invoked selection criterion of the maximal decay rate by showing that analytical, see Eq. (53), and numerical pushed TW solutions are essentially indistinguishable at various $\varphi$ and $N$ provided that $N>$ $\sqrt{1+\varphi^{2}}$. Finally, in all simulated cases such that $N>$ $\sqrt{1+\varphi^{2}}$, normalized fluxes $J$ asymptotically vanished up to the fourth digit with time, in line with the explicit analytical solution given by Eq. (53).

\section{CONCLUSIONS}

A telegraph-reaction-advection-diffusion equation was introduced by combining (i) the Maxwell-Cattaneo relaxation approach to describing diffusion and (ii) nonlinear advection term. The problem involves two governing nondimensional parameters, i.e., a ratio $\varphi^{2}$ of the relaxation time in the Maxwell-Cattaneo model to the characteristic time scale of the reaction term and the normalized magnitude $N$ of the advection term.

The introduced equation was linearized at the leading edge of the TW and the following analytical results summarized in Table I were derived. First, necessary conditions for the existence of pulled or pushed TW solutions that are smooth in the entire interval of $-\infty<\zeta<\infty$ were obtained. Second, the smooth TW speed was shown to be less than the maximal speed $\varphi^{-1}$ of the propagation of the considered substance. Third, the lowest TW speed as a function of $\varphi$ and $N$ was determined. Fourth, it has been shown that, if the necessary condition of $N>\varphi-\varphi^{-1}$ does not hold, e.g., if the magnitude of the advection term is insufficiently large in the case of $\varphi^{2}>1$, then the studied equation admits piecewise smooth TW solutions with sharp leading fronts that propagate at the maximal speed $\varphi^{-1}$, with the substance concentration or its spatial derivative jumping at the front. An increase in $N$ can make relevant TW solutions smooth.

In the case of $N>\varphi$, an explicit smooth pushed TW solution to the considered nonlinear equation was found in the entire interval of $-\infty<\zeta<\infty$.
By invoking a principle of the maximal decay rate of TW solution at its leading edge, relevant TW solutions were selected, as summarized in Table II. In particular, the transition from pulled to pushed smooth TW solutions was predicted to occur at $N=N_{\mathrm{cr}}=\sqrt{1+\varphi^{2}}$, with the pulled (pushed) TW being relevant at a smaller (larger) magnitude $N$ of the advection term. An increase in the normalized relaxation time $\varphi^{2}$ results in increasing $N_{\mathrm{cr}}$ and, therefore, can cause transition from pushed to pulled solutions.

An increase in the relaxation time reduces the lowest speed of the pulled relevant TW solutions, but affects neither the speed nor the structure of the pushed relevant TW solutions.

All the aforementioned analytical results and, in particular, the maximal-decay-rate principle or appearance of the piecewise smooth TW solutions, were validated by numerically solving the initial boundary value problem for the studied equation with natural initial conditions localized to a bounded spatial region.

\section{ACKNOWLEDGMENTS}

V.A.S. and N.N.P. gratefully acknowledge the financial support by ONERA. A.N.L. gratefully acknowledges the financial support by the Chalmers Transport and Energy Areas of Advance, and by the Combustion Engine Research Center (CERC).

\section{APPENDIX A: DISPERSION RELATION AT THE LEADING EDGE}

Substitution of Eq. (36) into Eqs. (34) and (35) yields the following homogeneous linear algebraic equations:

$$
\begin{aligned}
& \left(\frac{N-\Lambda \varphi^{2}}{\Lambda^{2} \varphi^{2}-1}+\kappa\right) U+\frac{\varphi^{-1}}{\Lambda^{2} \varphi^{2}-1} J=0 \\
& \frac{\Lambda \varphi N-\varphi}{\Lambda^{2} \varphi^{2}-1} U+\left(\frac{\Lambda}{\Lambda^{2} \varphi^{2}-1}+\kappa\right) J=0 .
\end{aligned}
$$

In order for a nontrivial solution to exist, the determinant of the system (A1) and (A2) has to be equal to zero, i.e.,

$$
\begin{gathered}
\left(\frac{N-\Lambda \varphi^{2}}{\Lambda^{2} \varphi^{2}-1}+\kappa\right)\left(\frac{\Lambda}{\Lambda^{2} \varphi^{2}-1}+\kappa\right) \\
-\frac{\Lambda \varphi N-\varphi}{\left(\Lambda^{2} \varphi^{2}-1\right)} \frac{\varphi^{-1}}{\left(\Lambda^{2} \varphi^{2}-1\right)}=0
\end{gathered}
$$


which reads

$$
\left(1-\Lambda^{2} \varphi^{2}\right) \kappa^{2}-\left(\Lambda\left(1-\varphi^{2}\right)+N_{B}\right) \kappa+1=0,
$$

after some algebra. Equation (A4) can also be obtained by substituting $u(\xi, \theta)=U(\zeta)$ and Eq. (36) into the reactiontelegraph Eq. (12).

The dispersion relation given by Eq. (A4) links the TW speed $\Lambda$ and the decay rate $\kappa>0$ of the profile $U(\zeta)$ at the leading edge and has two solutions:

$$
\begin{aligned}
& \kappa_{+}=\frac{\Lambda\left(1-\varphi^{2}\right)+N+\sqrt{\Delta_{0}(\varphi, \Lambda, N)}}{2\left(1-\Lambda^{2} \varphi^{2}\right)}, \\
& \kappa_{-}=\frac{\Lambda\left(1-\varphi^{2}\right)+N-\sqrt{\Delta_{0}(\varphi, \Lambda, N)}}{2\left(1-\Lambda^{2} \varphi^{2}\right)},
\end{aligned}
$$

such that

$$
\kappa_{+} \kappa_{-}=\frac{1}{1-\Lambda^{2} \varphi^{2}}
$$

where

$$
\Delta_{0}(\varphi, \Lambda, N)=\left(\Lambda\left(1-\varphi^{2}\right)+N\right)^{2}-4\left(1-\Lambda^{2} \varphi^{2}\right) \geqslant 0
$$

in order for $\kappa_{+}$and $\kappa_{-}$to be real. Equation (A7) shows that the two decay rates are of the same sign. Moreover, the decay rate should be positive in order for Eq. (36) to yield a finite $U(\zeta)$.

Thus the dispersion relation given by Eq. (A4) results in two constraints: (i) Eq. (A8), and (ii) $\kappa_{+}>0, \kappa_{-}>0$ by virtue of Eqs. (36) and (A7). Moreover, as discussed above, $\Lambda \geqslant \varphi^{-1}$.

First, let us consider consequences from the former constraint given by Eq. (A8). It can bound the TW speed from below, i.e.,

$$
\Lambda \geqslant \Lambda_{\min }(\varphi, N)>0,
$$

where $\Lambda_{\min }(\varphi, N)$ is found by solving the following quadratic equation:

$$
\begin{aligned}
\Delta_{0}\left(\varphi, \Lambda_{\min }, N\right)= & {\left[N-\left(\Lambda_{\min } \varphi\right)\left(\varphi-\varphi^{-1}\right)\right]^{2} } \\
& +4\left(\Lambda_{\min }^{2} \varphi^{2}-1\right)=0,
\end{aligned}
$$

which reads

$$
\left(\varphi+\varphi^{-1}\right)^{2}\left(\Lambda_{\min } \varphi\right)^{2}-2 N\left(\varphi-\varphi^{-1}\right)\left(\Lambda_{\min } \varphi\right)+N^{2}-4=0
$$

and is satisfied by

$$
\Lambda_{\min +}(\varphi, N) \varphi=\frac{2 N\left(\varphi-\varphi^{-1}\right)+\sqrt{\Delta_{0 \Lambda}(\varphi, N)}}{2\left(\varphi+\varphi^{-1}\right)^{2}},
$$

or

$$
\Lambda_{\min -}(\varphi, N) \varphi=\frac{2 N\left(\varphi-\varphi^{-1}\right)-\sqrt{\Delta_{0 \Lambda}(\varphi, N)}}{2\left(\varphi+\varphi^{-1}\right)^{2}},
$$

such that

$$
\Lambda_{\min +} \Lambda_{\min -} \varphi^{2}=\frac{N^{2}-4}{\left(\varphi+\varphi^{-1}\right)^{2}},
$$

where

$$
\begin{aligned}
\Delta_{0 \Lambda}(\varphi, N) & =4 N^{2}\left(\varphi-\varphi^{-1}\right)^{2}-4\left(\varphi+\varphi^{-1}\right)^{2}\left(N^{2}-4\right) \\
& =16\left[\left(\varphi+\varphi^{-1}\right)^{2}-N^{2}\right] .
\end{aligned}
$$

Because both $\Lambda_{\min }+$ and $\Lambda_{\min -}$ should be real,

$$
|N| \leqslant \varphi+\varphi^{-1} \text {. }
$$

Moreover, because $\Delta_{0}(\varphi, \Lambda, N)$ defined by Eq. (A8) is positive if either $\Lambda<\Lambda_{\min -}$ or $\Lambda>\Lambda_{\min +}$ and $\Lambda$ should be positive by virtue of Eq. (24), the TW speed can be bounded from below by $\Lambda_{\text {min }+}$ only. Accordingly, we will consider solely $\Lambda_{\text {min }+}$ in the following. Thus, we have to find a function $\Lambda_{\min +}(N, \varphi)$ in a range of $N$ and $\varphi$ such that (i) Eq. (A16) holds, (ii) $\Lambda_{\min +}>0$, and (iii) $\Lambda_{\min +} \varphi<1$.

If (a) $0<\varphi<1$, and, hence, $\varphi-\varphi^{-1}<0$, then, $\Lambda_{\min +}>$ 0 if $N<2$. Moreover, by virtue of Eq. (A16), $N$ should be bounded from below, i.e., $-\varphi-\varphi^{-1}<N<2$. In this interval, a constraint of $\Lambda_{\min } \varphi<1$ is also satisfied provided that $N \neq$ $\varphi-\varphi^{-1}$. If $N=\varphi-\varphi^{-1}$, then, $\Lambda_{\min +}=\varphi^{-1}$.

When $N$ is increased from $N=-\varphi-\varphi^{-1}$ to $N<\varphi-$ $\varphi^{-1}$, the product $\Lambda_{\min } \varphi$ is increased from

$$
\begin{aligned}
& \Lambda_{\min +}\left(\varphi<1, N=-\varphi-\varphi^{-1}\right) \varphi \\
& =\frac{-\left(\varphi+\varphi^{-1}\right)\left(\varphi-\varphi^{-1}\right)}{\left(\varphi+\varphi^{-1}\right)^{2}}=\frac{1-\varphi^{2}}{1+\varphi^{2}}<1
\end{aligned}
$$

to unity, i.e., $\Lambda_{\min +}\left(\varphi<1, N=\varphi-\varphi^{-1}\right) \varphi=1$. If $N$ is further increased and $\varphi-\varphi^{-1}<N<2$ then $\Lambda_{\min +} \varphi$ is decreased from unity to zero. Thus

$$
\Lambda_{\min +}(\varphi<1, N=2)=0 .
$$

If (b) $\varphi \geqslant 1$ and, hence, $\varphi-\varphi^{-1} \geqslant 0$, then, Eq. (A16) holds and $\Lambda_{\text {min }+}>0$ in the interval of $-2<N<\varphi+\varphi^{-1}$. Within this interval, the constraint of $\Lambda_{\min } \varphi<1$ is also satisfied provided that $N \neq \varphi-\varphi^{-1}$. If $N=\varphi-\varphi^{-1}$, then, $\Lambda_{\min +}=$ $\varphi^{-1}$.

When $N$ is increased from $N=-2$, the product $\Lambda_{\min +} \varphi$ is increased from zero, i.e.,

$$
\Lambda_{\min +}(\varphi>1, N=-2)=0,
$$

to unity at $N=\varphi-\varphi^{-1}$. If $N$ is further increased, but $\varphi-$ $\varphi^{-1}<N<\varphi+\varphi^{-1}$, then $\Lambda_{\min +} \varphi$ is decreased to

$$
\Lambda_{\min +}\left(\varphi>1, N=\varphi+\varphi^{-1}\right) \varphi=\frac{\varphi^{2}-1}{\varphi^{2}+1}<1 .
$$

In the particular case of $N=0$ (the lack of advection), we have

$$
\begin{aligned}
& \Lambda_{\min +}(\varphi, N=0) \varphi=\frac{2\left(\varphi+\varphi^{-1}\right)}{\left(\varphi+\varphi^{-1}\right)^{2}}=\frac{2 \varphi}{\varphi^{2}+1}<1 \text { or } \\
& \Lambda_{\min +}(1, N=0)=\frac{2}{\varphi^{2}+1}<1,
\end{aligned}
$$

if either $0<\varphi<1$ or $\varphi \geqslant 1$. This particular result was already obtained by Hadeler [35,36].

In the particular case of $\varphi=0$ (the lack of memory), we have

$$
\Lambda_{\min +}(\varphi=0, N)=-N+\sqrt{N^{2}-\left(N^{2}-4\right)}=-N+2,
$$

This particular result was obtained in [19-21].

In summary, Eq. (A8) shows that the TW speed can be bounded from zero and less than $\varphi^{-1}$ if $N \neq \varphi-\varphi^{-1}$ and either (a) $0<\varphi<1$ and $-\varphi-\varphi^{-1}<N<2$, or (b) $\varphi \geqslant 1$ and $-2<N<\varphi+\varphi^{-1}$. 
Second, let us consider consequences from a constraint that the decay rates given by Eqs. (A5) and (A6) should be positive, i.e., $\kappa_{+}>0$ and $\kappa_{-}>0$. As discussed in Sec. II C, we will restrict ourselves to the case of $\Lambda=\Lambda_{\min }>0$ and, therefore, $\kappa_{+}=\kappa_{-}=\kappa_{m}$. In this case, Eqs. (A5) and (A6) read

$$
\begin{aligned}
\kappa_{m} & =\kappa_{+}=\kappa_{-}=\frac{\Lambda_{\min }\left(1-\varphi^{2}\right)+N}{2\left(1-\varphi^{2} \Lambda_{\min }^{2}\right)} \\
& =\frac{\Lambda_{\min } \varphi\left(\varphi^{-1}-\varphi\right)+N}{2\left(1-\varphi^{2} \Lambda_{\min }^{2}\right)}=\frac{1}{\sqrt{\left(1-\varphi^{2} \Lambda_{\min }^{2}\right)}},
\end{aligned}
$$

because

$$
\begin{aligned}
& N-\left(\Lambda_{\min } \varphi\right)\left(\varphi-\varphi^{-1}\right)=2 \sqrt{\left(1-\Lambda_{\min }^{2} \varphi^{2}\right)} \\
& \text { if } \quad N-\left(\Lambda_{\min } \varphi\right)\left(\varphi-\varphi^{-1}\right)>0
\end{aligned}
$$

by virtue of Eq. (A10). It is worth noting that the decay rate tends to infinity, i.e., $\kappa_{m} \rightarrow \infty$, if $N \rightarrow\left(\varphi-\varphi^{-1}\right)+$ 0 , because, as shown above, $\Lambda_{\min +} \varphi \rightarrow 1-0$ as $N \rightarrow$ $\left(\varphi-\varphi^{-1}\right)+0$.

The decay rate is positive, i.e., $\kappa_{m}>0$, if the following two inequalities hold:

$$
\Lambda_{\min } \varphi<1, \quad N-\left(\Lambda_{\min } \varphi\right)\left(\varphi-\varphi^{-1}\right)>0 .
$$

Using the previous analysis of Eq. (A10), one can easily show that $\kappa_{m}>0$ if either $0<\varphi<1$ and $\varphi-\varphi^{-1}<N<2$ or $\varphi \geqslant 1$ and $\varphi-\varphi^{-1}<N<\varphi+\varphi^{-1}$. These two intervals are reduced when compared to intervals obtained earlier by analyzing Eq. (A8), i.e., $-\varphi-\varphi^{-1}<N<2$ if $0<\varphi<1$ or $-2<N<\varphi+\varphi^{-1}$ if $\varphi \geqslant 1$.

\section{APPENDIX B: TWS WITH MAXIMAL PROPAGATION SPEED, $\Lambda=\varphi^{-1}$}

By direct substitution, one can easily verify that

$$
U^{m}(1-U)^{n}=U_{0}^{m}\left(1-U_{0}\right)^{n} \exp \left[\varphi\left(\zeta-\zeta_{0}\right)\right]
$$

gives an implicit expression for a general solution to Eq. (44). Here, $\zeta_{0}$ is an arbitrary constant, $U_{0}=U\left(\zeta_{0}\right)$, and the power exponents $m$ and $n$ are as follows:

$$
m=\varphi^{2}-1-N \varphi, \quad n=m+2 .
$$

Due to invariance of Eq. (44) with respect to shift in space, we can set $\zeta_{0}=0$ without loss of generality.

The solution given by Eq. (B1) is not bounded for $-\infty<$ $\zeta<\infty$ with the exception of case (iii); see a list of five different cases in Sec. III B. Let us show that, in the four other cases, solutions to the considered BVP are piecewise smooth functions obtained by combining (a) a homogeneous state of $U=1$ or $U=0$, (b) a jump discontinuity of $U$ itself or its derivative $d U / d \zeta$, and (c) Eq. (B1), which is bounded in a semi-infinite interval of $\zeta_{0}<\zeta<\infty$ or $-\infty<\zeta<\zeta_{0}$.

\section{Piecewise smooth TW with jump discontinuity at the sharp leading front}

In the case of $N<\varphi-\varphi^{-1}$, see item (i) in Sec. III B, we have $m=\varphi^{2}-1-N \varphi>0$ and Eq. (44) has a singularity, because $a\left(U_{*} ; \varphi, N\right)=0$, where $U_{*}$ is given by Eq. (46). The coefficient $a(U ; \varphi, N)$ defined by Eq. (45) is negative at $0<$
$U<U_{*}$ and positive at $U_{*}<U<1$. The derivative $d U / d \zeta$ changes its sign at $U=U_{*}$ and is unbounded at $U=U_{*}$. Thus, if a solution to Eq. (44) exists in this case, the solution cannot be smooth over the entire interval of $-\infty<\zeta<\infty$, but has to be discontinuous. Let us assume that the functions $U$ and $J$ jump at the right sharp front $\xi_{r}(\theta)=\xi_{20}+\varphi^{-1} \theta$, i.e., we seek a piecewise discontinuous TW solution. If $\zeta_{0}$ is a position of the jump, then $U_{L}=U\left(\zeta_{0}-\right)$ and $J_{L}=J\left(\zeta_{0}-\right)$ are values of the concentration and the flux just behind (on the left) of the jump, while $U_{R}=U\left(\zeta_{0}+\right)$ and $J_{R}=J\left(\zeta_{0}+\right)$ are values of the concentration and the flux just ahead (on the right) of the jump. In an interval of $-\infty<\zeta<\zeta_{0}$, a smooth solution connects $U(-\infty)=1$ and $J(-\infty)=0$ with $U_{L}$ and $J_{L}$. In an interval of $\zeta_{0}<\zeta<\infty$, the solution is also smooth, because, according to Eq. (20), the concentration and flux vanish ahead of the jump. Therefore, $U_{L}$ and $J_{L}$ are the magnitudes of the jumps in $U$ and $J$ at $\zeta_{0}$, i.e., $U\left(\zeta_{0}-\right)-U\left(\zeta_{0}+\right)=U_{L}$ and $J\left(\zeta_{0}-\right)-J\left(\zeta_{0}+\right)=J_{L}$.

Integration of Eq. (30) from $\zeta_{0}-0$ to $\zeta_{0}+0$ yields

$$
-\left(U_{L}-U_{R}\right)+\left(J_{L}-J_{R}\right)=\varphi \int_{-0}^{+0} U(1-U) d \xi=0,
$$

because the integrand is bounded ( $U$ is a bounded function), and the integration interval is infinitesimal. As

$$
U_{R}=U\left(\zeta_{0}+\right)=0, \quad J_{R}=J\left(\zeta_{0}+\right)=0,
$$

Eq. (B3) reduces to

$$
J_{L}=U_{L}
$$

Substitution of Eq. (B5) into Eq. (33) yields $U_{L}=$ $(m+1) U_{L}\left(1-U_{L}\right)$, where $m=\varphi^{2}-1-N \varphi>0$. Consequently,

$$
U_{L}=U\left(\zeta_{0}-\right)=2 U_{*}=\frac{m}{m+1}<1, \quad 0<U_{L}<1 .
$$

Comparison of Eqs. (46) and (B6) shows that $U_{*}=U_{L} / 2<$ $U_{L}$. Therefore, the coefficient $a(U ; \varphi, N)$ is positive and $d U / d \zeta<0$ in a region of $-\infty<\zeta<0$ where $U_{L}<U<1$, i.e., the discussed solution $U(\zeta)$ to Eq. (44) monotonously decreases from unity at the left boundary $\zeta=-\infty$ to $U_{L}$ at $\zeta=0$.

It is worth noting that $U_{L}=0$ if $N=\varphi-\varphi^{-1}$ and $U_{L} \rightarrow 1$ if $N \rightarrow-\infty$ at a constant $\varphi$, while the case of $N \rightarrow \infty$ is not considered, because $N<\varphi-\varphi^{-1}$, as stated in the beginning of the present subsection.

In a semi-interval of $-\infty<\zeta<0$ (here, $\zeta_{0}=0$ for simplicity), a solution to Eq. (44) is given by Eqs. (B1) and (B2) where $U_{0}=U_{L}$ in order to satisfy the boundary condition of $U(0-)=U_{L}$. Therefore, the solution reads

$$
\begin{aligned}
U^{m}(1-U)^{n} & =U_{L}{ }^{m}\left(1-U_{L}\right)^{n} \exp (\varphi \zeta), \\
n & =m+2>0, \quad \zeta<0 .
\end{aligned}
$$

To conclude this subsection, let us obtain Eq. (B6) using another method. First, let us rewrite Eq. (44) in another, "conservative" form,

$$
\frac{d F}{d \zeta}=-\varphi U(1-U)
$$


where the function $F$ (the generalized total flux) is given by

$$
F=[1-(m+1)(1-U)] U .
$$

Equations (44) and (B8) and (B9) are equivalent to one another for a smooth function. However, contrary to Eq. (44), Eqs. (B8) and (B9) can be used for discontinuous solutions, because the function $F$ is continuous even for waves that are discontinuous at $\zeta_{0}$. Indeed, integration of Eq. (B8) over a small interval $\Delta \zeta$ around $\zeta_{0}$ yields

$$
\begin{aligned}
& F\left(\zeta_{0}+\frac{\Delta \zeta}{2}\right)-F\left(\zeta_{0}-\frac{\Delta \zeta}{2}\right) \\
& \quad=-\int_{\zeta_{0}-\Delta \zeta / 2}^{\zeta_{0}+\Delta \zeta / 2} \varphi U(1-U) d \zeta \rightarrow 0, \text { if } \Delta \zeta \rightarrow 0 .
\end{aligned}
$$

The function $F$ vanishes at $\zeta_{0}+0$, i.e., $F\left(\zeta_{0}+0\right)=0$, due to Eq. (B4). Then, the continuity of the total flux yields

$$
F\left(\zeta_{0}-0\right)=F\left(\zeta_{0}+0\right)=U_{L}\left[1-(m+1)\left(1-U_{L}\right)\right]=0,
$$

thus resulting in Eq. (B6).

\section{Piecewise smooth TW with discontinuous derivatives at the sharp leading front}

In the case of $N=\varphi-\varphi^{-1}$, see item (ii) in Sec. III B, Eqs. (B1) and (B2) are consistent with the boundary condition given by Eq. (27) only if the TW solution is smooth in a semiinterval of $\zeta \leqslant 0$ and $U(\zeta)=0$ at $\zeta>0$, i.e., the TW solution has discontinuous derivative at the sharp leading front. In other words, the TW solution is a piecewise smooth function. In the considered case, $m=0, n=2$, and Eq. (B1) reads

$$
(1-U)^{2}=\exp (\varphi \zeta), \zeta \leqslant 0 .
$$

Equation (B12) satisfies both Eq. (44) and boundary conditions of $U(-\infty)=1$ and $U(0)=0$. Therefore, the piecewise smooth TW solution is as follows:

$$
U(\zeta)=\left\{\begin{array}{ll}
1-\exp \left(\frac{\varphi}{2} \zeta\right) & \text { if } \zeta \leqslant 0 \\
0 & \text { if } \zeta>0
\end{array} .\right.
$$

\section{Smooth TWs in the entire interval of $-\infty<\zeta<\infty$}

In the case of $\varphi-\varphi^{-1}<N<\varphi+\varphi^{-1}$, see item (iii) in Sec. III B, we have $-2<m<0,0<n<2$, and the boundary conditions given by Eq. (27) are satisfied. If $U_{0}=1 / 2$, then, Eqs. (B1) and (B2) read

$$
\begin{aligned}
U^{m}(1-U)^{n} & =2^{-(m+n)} \exp \left[\varphi\left(\zeta-\zeta_{0}\right)\right], \\
m+n & =2(m+1)=2\left(\varphi^{2}-N \varphi\right), \\
-2 & <m+n<2 .
\end{aligned}
$$

The asymptotic behavior of the solution is as follows:

$$
\begin{array}{r}
U \propto \exp \left[-\left(N-\varphi+\varphi^{-1}\right)^{-1} \zeta\right] \rightarrow 0, \quad \zeta \rightarrow \infty \\
1-U \propto \exp \left[\left(\varphi+\varphi^{-1}-N\right)^{-1} \zeta\right] \rightarrow 0, \quad \zeta \rightarrow-\infty
\end{array}
$$

At the leading edge, the decay rate of the TW solution is equal to $\left(N-\varphi+\varphi^{-1}\right)^{-1}$; see Eq. (B14). The same decay rate results from substitution of $\Lambda \varphi=1$ into the dispersion relation (A4), which reads

$$
-\left(N-\varphi+\varphi^{-1}\right) \kappa+1=0
$$

in this case.

The flux $J$ is given by Eq. (33), which yields $J>0$ if $\varphi-\varphi^{-1}<N<\varphi, J=0$ if $N=\varphi$, and $J<0$ if $\varphi<N<$ $\varphi+\varphi^{-1}$.

As discussed in Sec. III B, the smooth TW solution obtained at $\Lambda \varphi=1$ is not relevant.

\section{Piecewise smooth TW with discontinuous derivatives at the trailing edge}

In the case of $N=\varphi+\varphi^{-1}$, see item (iv) in Sec. III B, Eqs. (B1) and (B2) can be consistent with the boundary condition given by Eq. (27) only if the TW solution is smooth in a semi-interval of $\zeta \geqslant 0$ and $U(\zeta)=1$ at $\zeta<0$, i.e., the TW solution has discontinuous derivative at the trailing edge. In other words, the TW solution is a piecewise smooth function. In the considered case, $m=-2, n=0$, and Eq. (B1) reads

$$
U^{-2}=\exp (\varphi \zeta), \quad \zeta \geqslant 0 .
$$

Equation (B17) satisfies both Eq. (44) and boundary conditions of $U(0)=1$ and $U(\infty)=0$. Therefore, the piecewise smooth TW solution is as follows:

$$
U(\zeta)=\left\{\begin{array}{ll}
1 & \text { if } \zeta<0 \\
\exp \left(-\frac{\varphi}{2} \zeta\right) & \text { if } \zeta \geqslant 0
\end{array} .\right.
$$

Substitution of $N=\varphi+\varphi^{-1}$ into Eq. (33) yields the following normalized flux:

$$
J=-U(1-U)<0, \quad \zeta \geqslant 0 .
$$

The obtained piecewise smooth TW solution with $\Lambda \varphi=1$ is not relevant; see Sec. III B.

\section{Piecewise smooth TW with jump discontinuity at the trailing edge}

In the case of $N>\varphi+\varphi^{-1}$, see item (v) in Sec. III B, we have $m=\varphi^{2}-1-N \varphi<-2$ and, similarly to case (i), Eq. (44) has a singularity, because $a\left(U_{*} ; \varphi, N\right)=0$, where $U_{*}$ is given by Eq. (46). Repeating the analysis done in subsection 1 of the present Appendix, we arrive at the following results.

The functions $U$ and $J$ jump at the trailing edge. If $\zeta_{0}$ is a position of the jump, then, in a semi-interval of $-\infty<\zeta<\zeta_{0}$, the concentration and flux are equal to 1 and $0, U_{L}=U\left(\zeta_{0}-\right)=1, J_{L}=J\left(\zeta_{0}-\right)=0$, respectively. In a semi-interval of $\zeta_{0}<\zeta<\infty$, a smooth solution given by (B1) connects $U_{R}=U\left(\zeta_{0}+\right)$ and $J_{R}=J\left(\zeta_{0}+\right)$ with $U(-\infty)=1$ and $J(-\infty)=0$. Therefore, the magnitudes of the jumps in $U$ and $J$ at $\zeta_{0}$ are equal to $U\left(\zeta_{0}-\right)-U\left(\zeta_{0}+\right)=1-U_{R}$ and $J\left(\zeta_{0}-\right)-J\left(\zeta_{0}+\right)=-J_{R}$.

Integration of Eq. (30) from $\zeta_{0}-0$ to $\zeta_{0}+0$ yields

$$
-\left(U_{L}-U_{R}\right)+\left(J_{L}-J_{R}\right)=0 .
$$

Because

$$
U_{L}=U\left(\zeta_{0}-\right)=1, \quad J_{L}=J\left(\zeta_{0}-\right)=0,
$$

Eq. (B20) reduces to

$$
-1+U_{R}-J_{R}=0, \quad J_{R}=U_{R}-1 .
$$


Substitution of Eq. (B22) into Eq. (33) yields $U_{R}-1=$ $(m+1) U_{R}\left(1-U_{R}\right)$, where $m=\varphi^{2}-1-N \varphi<-2$. Consequently,

$$
\begin{aligned}
& U_{R}=U\left(\zeta_{0}+\right)=-\frac{1}{m+1}, \\
& 0<U_{R}<1, \quad J_{R}=-\frac{m+2}{m+1}<0 .
\end{aligned}
$$

Comparison of Eqs. (46) and (B23) shows that

$$
U_{*}-U_{R}=\frac{m}{2(m+1)}+\frac{1}{m+1}=\frac{m+2}{m+1}>0 .
$$

Therefore, the coefficient $a(U ; \varphi, N)$ is positive and $d U / d \zeta<0$ in a region of $\zeta_{0}<\zeta<\infty$ where $0<U<U_{R}$. In a semi-interval of $0<\zeta<\infty\left(\zeta_{0}=0\right)$, a solution to Eq. (44) is given by Eqs. (B1) and (B2) where $U_{0}=U_{R}$. Therefore, the implicit solution reads

$$
U^{m}(1-U)^{n}=U_{R}^{m}\left(1-U_{R}\right)^{n} \exp (\varphi \zeta), \quad 0<\zeta<\infty .
$$

The solution $U(\zeta)$ given by Eq. (B24) monotonously decreases from $U_{R}$ at $\zeta_{0}+$ to zero at $\zeta=\infty$. The asymptotic behavior of the solution at the leading edge is as follows:

$$
\begin{aligned}
U & \rightarrow 1-U_{R}^{m / n}\left(1-U_{R}\right) \exp \left[\left(\varphi+\varphi^{-1}-N\right)^{-1} \zeta\right] \rightarrow 0, \\
\zeta & \rightarrow \infty .
\end{aligned}
$$

The obtained piecewise smooth TW solution with $\Lambda \varphi=1$ is not relevant, see Sec. III B.

\section{APPENDIX C: NUMERICAL SCHEME}

A numerical solution to PDEs (13) and (14) with $Q=$ $u(1-u)$ and $\omega=u(1-u)$ was obtained using a finite volume scheme for the advection terms in these equations, e.g., see [49]. A finite space-time rectangle domain of $\left(a_{l}, a_{r}\right) \times(0, \theta)$ was discretized using a uniform grid with the mesh width $\Delta \xi$ and the constant time step $\Delta \theta$. Here, $a_{l}=-150$ and $a_{r}=150$ are the left and right boundaries of the spatial subdomain, cell centers $\xi_{i}=a_{l}+(i-1) \Delta \xi$ and instants $\theta_{n}=n \Delta \theta$ constitute discrete mesh points $\left(\xi_{i}, \theta_{n}\right), 1 \leqslant i \leqslant I$ and $0 \leqslant n$, the mesh step $\Delta \xi=\left(a_{r}-a_{l}\right) /(I-1), I=48000$. Obtained numerical approximations of solutions to PDEs (13) and (14) will be denoted with $u_{+}^{i ; n}$ and $u_{-}^{i ; n}$, respectively.

To avoid spurious oscillations (wiggles) when calculating the piecewise smooth (nondifferentiable and even discontinuous) TW solutions, and to minimize the impact of numerical diffusion, the total variation diminishing (TVD) nonlinear flux-limiter scheme [46,49] was used. For smooth solutions, the scheme has the second-order accuracy. The source terms in PDEs (13) and (14) were discretized using the Euler explicit method. Discretized equations read

$$
\begin{aligned}
u_{+}^{i, n+1}= & u_{+}^{i, n}-\frac{\Delta \theta}{\Delta \xi} \varphi^{-1}\left(u_{+}^{i, n}+\frac{\Delta \xi}{2} \sigma_{+}^{i, n}-u_{+}^{i-1, n}\right. \\
& \left.-\frac{\Delta \xi}{2} \sigma_{+}^{i-1, n}\right)+\Delta \theta S_{+}^{i, n} \\
u_{-}^{i, n+1}= & u_{-}^{i, n}+\frac{\Delta \theta}{\Delta \xi} \varphi^{-1}\left(u_{-}^{i+1, n}-\frac{\Delta \xi}{2} \sigma_{+}^{i+1, n}\right. \\
& \left.-u_{-}^{i, n}+\frac{\Delta \xi}{2} \sigma_{-}^{i, n}\right)+\Delta \theta S_{-}^{i, n} .
\end{aligned}
$$

Here,

$$
\begin{aligned}
S_{+}^{i, n}= & \frac{1}{2} \varphi^{-2}\left(u_{-}^{i, n}-u_{+}^{i, n}\right)+\frac{1}{2}\left(1-\varphi^{-1} N\right) \\
& \times\left(u_{+}^{i, n}+u_{-}^{i, n}\right)\left(1-u_{+}^{i, n}-u_{-}^{i, n}\right), \\
S_{-}^{i, n}= & -\frac{1}{2} \varphi^{-2}\left(u_{-}^{i, n}-u_{+}^{i, n}\right)+\frac{1}{2}\left(1+\varphi^{-1} N\right) \\
& \times\left(u_{+}^{i, n}+u_{-}^{i, n}\right)\left(1-u_{+}^{i, n}-u_{-}^{i, n}\right),
\end{aligned}
$$

and $\sigma_{+}^{i, n}$ and $\sigma_{-}^{i, n}$ stand for the nonlinear minmod slopes,

$$
\begin{aligned}
\sigma_{+}^{i, n} & =\operatorname{minmod}\left(\frac{u_{+}^{i, n}-u_{+}^{i-1, n}}{\Delta \xi}, \frac{u_{+}^{i+1, n}-u_{+}^{i, n}}{\Delta \xi}\right), \\
\sigma_{-}^{i 1, n} & =\min \bmod \left(\frac{u_{-}^{i, n}-u_{-}^{i-1, n}}{\Delta \xi}, \frac{u_{-}^{i+1, n}-u_{-}^{i, n}}{\Delta \xi}\right),
\end{aligned}
$$

where

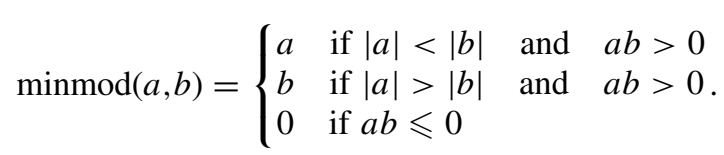

To keep the numerical process stable, the time step was chosen to satisfy Courant-Friedrichs-Levy (CFL) condition, i.e., $\Delta \theta<\operatorname{CFL} \varphi \Delta \xi$, where $\mathrm{CFL}<1$. The present calculations were carried out with CFL $=0.1$. As stressed in Sec. I, a limit of $\varphi \rightarrow+0$ (very short memory) is singular, because the reaction telegraph Eq. (12) reduces to the diffusion equation in this limit case. Accordingly, the present simulations were performed at $\varphi \geqslant 0.5$.

The initial conditions are given by Eqs. (60) and (61) or (62) and (63) for step and ramp, respectively. The boundary conditions are given by Eq. (64).

To check the convergence of a numerical solution to an asymptotic TW, we tracked points $\xi_{C}(\theta)$, where $u\left(\xi_{C}(\theta), \theta\right)=$ $C$. Then, the speeds $d \xi_{C} / d \theta$ of these points were calculated. Five values of $C$ were taken, i.e., $C=1 / 8,1 / 4,1 / 2,3 / 4$, and $7 / 8$. The numerical solution $u^{n}$ was assumed to converge to the asymptotic TW solution when the speeds of the five level points coincided up to the fourth digit. In our calculations, this condition was fulfilled when the simulation time (transient time) was larger than 100 , i.e., $n \times \Delta \theta>100$.
[1] R. A. Fisher, Ann. Eugenics 7, 355 (1937).

[2] A. N. Kolmogorov, E. G. Petrovsky, and N. S. Piskounov, Bulleten MGU, Moscow State University, USSR, Mathematics and Mechanics, International Ser. A1 No. 6, 1 (1937).
[3] J. D. Murray, Lectures on Nonlinear-Differential-Equation Models in Biology (Clarendon, Oxford, UK, 1977).

[4] J. D. Murray, Mathematical Biology 1. An Introduction (Springer, Berlin, 2002). 
[5] N. F. Britton, Reaction-Diffusion Equations and Their Applications to Biology (Academic Press, London, 1986).

[6] D. G. Aronson and H. F. Weinberger, in Partial Differential Equations and Related Topics, edited byJ. A. Goldstein, Lecture Notes in Mathematics, Vol. 446 (Springer, Berlin, 1975), p. 5.

[7] Ya. B. Zeldovich, G. I. Barenblatt, V. B. Librovich, and G. M. Makhviladze, The Mathematical Theory of Combustion and Explosions (Plenum, New York, 1985).

[8] A. G. Merzhanov and E. N. Rumanov, Rev. Mod. Phys. 71, 1173 (1999).

[9] R. J. Field and M. Burger, Oscillations and Traveling Waves in Chemical Systems (Wiley Interscience, New York, 1985).

[10] B. Straughan, Heat Waves, Applied Mathematics Science Series Vol. 177 (Springer, Berlin, 2011).

[11] M. C. Cross and P. C Hohenberg, Rev. Mod. Phys. 65, 851 (1993).

[12] W. van Saarloos, Phys. Rep. 386, 29 (2003).

[13] J. D. Logan, Transport Modeling in Hydrogeochemical Systems, Interdisciplinary Applied Mathematics Vol. 15 (Springer, New York, 2001).

[14] P. Grindrod, The Theory and Applications of Reaction-Diffusion Equations (Oxford University Press, New York, 1996).

[15] O. M. Phillips, Flow and Reactions in Permeable Rocks (Cambridge University Press, Cambridge, UK, 1991).

[16] V. Méndez, S. Fedotov, and W. Horsthemke, Reaction-Transport Systems: Mesoscopic Foundations, Fronts, and Spatial Instabilities (Springer, Berlin, 2010).

[17] F. S. Berezovskaya and G. P. Karev, Phys. Usp. 42, 917 (1999).

[18] D. Veynante, A. Trouvé, K. N. C. Bray, and T. Mantel, J. Fluid Mech. 332, 263 (1997).

[19] V. A. Sabelnikov and A. N. Lipatnikov, Combust. Theory Modell. 17, 1154 (2013).

[20] V. A. Sabelnikov and A. N. Lipatnikov, Phys. Rev. E 90, 033004 (2014).

[21] V. A. Sabelnikov and A. N. Lipatnikov, Combust. Flame 162, 2893 (2015).

[22] K. N. C. Bray, Proc. R. Soc. London, Ser. A 451, 231 (1995).

[23] A. N. Lipatnikov and J. Chomiak, Prog. Energy Combust. Sci. 36, 1 (2010).

[24] V. A. Sabelnikov and A. N. Lipatnikov, Annu. Rev. Fluid Mech. 49 (2016), doi:10.1146/annurev-fluid-010816-060104
[25] A.C. Scurlock and J.H. Grover, Symp. (Int.) Combust. 4, 645 (1953).

[26] P. A. Libby and K. N. C. Bray, AIAA J. 19, 205 (1981).

[27] E. Zauderer, Partial Differential Equations of Applied Mathematics (Wiley Interscience, New York, 2006).

[28] D. D. Joseph and L. Preziosi, Rev. Mod. Phys. 61, 41 (1989).

[29] J. C. Maxwell, Phil. Trans. R. Soc. London 157, 49 (1867).

[30] C. Cattaneo, Atti del Semin. Mat. e Fis. Univ. Modena 3, 3 (1948).

[31] C. Cattaneo, C. R. Acad. Sci. 247, 431 (1958).

[32] U. Ebert and W. van Saarloos, Physica D 146, 1 (2000).

[33] B. I. Davydov, Dokl. Akad. Nauk SSSR 2(7), 474 (1935).

[34] V. Méndez, D. Campos, and W. Horsthemke, Phys. Rev. E 90, 042114 (2014).

[35] K. P. Hadeler, Can. Appl. Math. Quart. 2, 27 (1994).

[36] K. P. Hadeler, in Stochastic and Spatial Structures of Dynamical Systems, edited by S. J. van Strien and S. M. Verduyn Lunal (Royal Netherlands Academy of Arts and Sciences, 1996), p. 133.

[37] P. M. Jordan, Physica D, 207, 220 (2005).

[38] A. S. Monin and A. M. Yaglom, Statistical Fluid Mechanics (MIT Press, Cambridge, MA, 1971), Vol. I.

[39] V. L. Zimont and V. A. Sabelnikov, Izv., Acad. Sci., USSR, Atmos. Oceanic Phys. (Engl. Transl.) 1, 385 (1975).

[40] J. D. Logan, An Introduction to Nonlinear Partial Differential Equations (Wiley Interscience, New York, 2008).

[41] S. Puri, K. R. Elder, and R. C. Desai, Phys. Lett. A 142, 357 (1989).

[42] S. Puri, Phys. Rev. A 43, 7031 (1991).

[43] G. T. Dee and W. van Saarloos, Phys. Rev. Lett. 60, 2641 (1988).

[44] W. van Saarloos, Phys. Rev. A 37, 211 (1988).

[45] W. van Saarloos, Phys. Rev. A 39, 6367 (1989).

[46] E. Bouin, V. Calvez, and G. Nadin, Math. Models Methods Appl. Sci. 24, 1165 (2014).

[47] P. L. Sachdev and Ch. Srinivasa Rao, Large Time Asymptotics for Solutions of Nonlinear Partial Differential Equations (Springer Science \& Business Media, New York, 2009).

[48] G. I. Barenblatt, Scaling, Self-Similarity, and Intermediate Asymptotics (Cambridge University Press, Cambridge, UK, 1996).

[49] R. J. Le Veque, Finite Volume Methods for Hyperbolic Problems (Cambridge University Press, New York, 2002). 